OPEN ACCESS

Edited by:

Isabel Lastres-Becker, Autonomous University of Madrid,

Spain

Reviewed by:

Kunie Ando,

Université libre de Bruxelles, Belgium

Charles Robert Harrington,

University of Aberdeen,

United Kingdom

*Correspondence: Kiran Bhaskar

KBhaskar@salud.unm.edu

Received: 23 July 2020 Accepted: 24 September 2020

Published: 16 October 2020

Citation:

Jiang S and Bhaskar K (2020) Degradation and Transmission of Tau

by Autophagic-Endolysosomal Networks and Potential Therapeutic

Targets for Tauopathy.

Front. Mol. Neurosci. 13:586731. doi: $10.3389 /$ fnmol.2020.586731

\section{Degradation and Transmission of Tau by Autophagic-Endolysosomal Networks and Potential Therapeutic Targets for Tauopathy}

\author{
Shanya Jiang and Kiran Bhaskar* \\ Department of Molecular Genetics and Microbiology, The University of New Mexico, Albuquerque, NM, United States
}

Tauopathies are a class of neurodegenerative diseases, including Alzheimer's disease (AD), Frontotemporal Dementia (FTD), Progressive Supranuclear Palsy (PSP), Corticobasal Degeneration (CBD), and many others where microtubule-associated protein tau (MAPT or tau) is hyperphosphorylated and aggregated to form insoluble paired helical filaments (PHFs) and ultimately neurofibrillary tangles (NFTs). Autophagicendolysosomal networks (AELN) play important roles in tau clearance. Excessive soluble neurotoxic forms of tau and tau hyperphosphorylated at specific sites are cleared through the ubiquitin-proteasome system (UPS), Chaperon-mediated Autophagy (CMA), and endosomal microautophagy (e-MI). On the other hand, intra-neuronal insoluble tau aggregates are often degraded within lysosomes by macroautophagy. AELN defects have been observed in AD, FTD, CBD, and PSP, and lysosomal dysfunction was shown to promote the cleavage and neurotoxicity of tau. Moreover, several AD risk genes (e.g., PICALM, GRN, and BIN1) have been associated with dysregulation of AELN in the late-onset sporadic AD. Conversely, tau dissociation from microtubules interferes with retrograde transport of autophagosomes to lysosomes, and that tau fragments can also lead to lysosomal dysfunction. Recent studies suggest that tau is not merely an intraneuronal protein, but it can be released to brain parenchyma via extracellular vesicles, like exosomes and ectosomes, and thus spread between neurons. Extracellular tau can also be taken up by microglial cells and astrocytes, either being degraded through AELN or propagated via exosomes. This article reviews the complex roles of AELN in the degradation and transmission of tau, potential diagnostic/therapeutic targets and strategies based on AELN-mediated tau clearance and propagation, and the current state of drug development targeting AELN and tau against tauopathies.

Keywords: tauopathy, degradation, transmission, tau, neuron, glial cells, autophagy, endo-lysosomal systems

\section{INTRODUCTION}

Microtubule-associated protein tau (MAPT or tau) is a neuronal protein, which binds to the microtubule and regulates its assembly and stabilization, thus mediating the axonal transport of cellular components along the microtubule. Tau is constantly phosphorylated and dephosphorylated under physiological conditions (Lee et al., 2001; Götz et al., 2019). However, 
under pathological conditions, tau undergoes various posttranslational modifications and detaches from microtubule to form insoluble aggregates, named paired helical filaments (PHFs) that ultimately grow into neurofibrillary tangles (NFTs).

A variety of post-translational modifications and aggregation of tau and the presence of PHFs and NFTs inside neurons or glial cells (astrocytes and oligodendrocytes) is the pathological hallmark of AD and related tauopathies (Lee et al., 2001). Tau pathology positively correlates with the disease progression and aligns well with Braak staging (Schöll et al., 2016). Different tauopathies display different brain regional susceptibility and distinct clinical symptoms due to the underlying type and extent of tau pathologies (Götz et al., 2019).

The Ubiquitin-proteasome system (UPS) and autophagy are two major intracellular degradation process (Dikic, 2017). UPS mainly degrades short-lived proteins, and autophagy is responsible for the clearance of long-lived proteins and damaged organelles. Autophagy can be further categorized into chaperonmediated autophagy (CMA), endosomal microautophagy (e-MI), and macroautophagy.

Soluble neurotoxic tau is cleared by UPS (Lee et al., 2013), CMA (Wang et al., 2009), and e-MI (Caballero et al., 2018; Uytterhoeven et al., 2018), while intracellular insoluble tau is degraded by macroautophagy (Krüger et al., 2012). In addition, tau phosphorylated at specific sites can also be removed by the autophagy-independent endolysosomal system (Vaz-Silva et al., 2018). Apart from AD/tauopathies, NFTs are also found in brains of subjects with Niemann-Pick type C (NPC), a lysosomal storage disease resulting from lysosomal function defects (Wang and Mandelkow, 2012). Thus, it suggests the Autophagy Endo-Lysosomal Network (AELN) plays an important role in preventing tau aggregation and NFT accumulation in different tauopathies and lysosomal storage diseases.

Indeed, accumulation of lysosomes, lysosomal hydrolases, autophagosome, autophagic vacuoles, multi-vesicular bodies (MVBs), autolysosomes, and defect in lysosomal membrane integrity in the brain has been reported in $\mathrm{AD}, \mathrm{CBD}$, and PSP patients (Nixon et al., 2005; Nixon, 2013; Piras et al., 2016; Menzies et al., 2017). Defects in the retrograde transportation of autophagosomes from axon to soma and antegrade transportation of degradative lysosomes to distal axons were also demonstrated (Farfel-Becker et al., 2019). On the other hand, fragmented tau can impair CMA and lysosomal dysfunction and lead to tau oligomerization and aggregation (Wang et al., 2009).

BIN1, PICALM, CD2AP, RIN3, SORL1, GRN, and PLD3, which affect AELN functions (Van Acker et al., 2019), were identified as AD susceptibility loci in genome-wide association studies (reviewed in Van Acker et al., 2019). Particularly, BIN1, PICALM, and GRN have been implicated in tauopathies. These linkage studies suggest the failure of AELN can contribute to tau pathology in tauopathies and related diseases.

Interestingly, AELN is also involved in the propagation of tau. Physiological and pathological tau can both be secreted from neurons through exosomes, the extracellular vesicles contained inside MVBs (an intermediate endocytic compartment) (Saman et al., 2011; Wang et al., 2017). MVB fusion with the plasma membrane releases exosomes to the extracellular space. Secretory autophagy-based unconventional secretion of pathological tau has also been reported in a recent study (Kang et al., 2019).

Uptake of different forms of tau by neurons utilizes AELN as well. Internalized tau then clogs up UPS and AELN, further inhibiting tau degradation and contributing to pathological tau accumulation (Wu et al., 2013). In addition, once internalized, tau aggregates can rupture endosomes to seed more tau aggregates and propagate in the recipient cells (Calafate et al., 2016).

Microglia can effectively phagocytose tau aggregates bound to anti-tau antibodies and degrade them through AELN (Luo et al., 2015). On the other hand, microglia can also be a foe in propagating tau pathology by packaging tau into exosome and release into extracellular space (Asai et al., 2015). Furthermore, astrocytes can take up tau fibrils through HSPG-mediated micropinocytosis (Martini-Stoica et al., 2018) and tau monomers through an HSPG-independent pathway (Perea et al., 2019). Upon internalization into astrocytes, astroglial transcription factor - EB (TFEB, a master regular of autophagy) promotes tau degradation and the inhibition of tau transmission in tauopathies (Martini-Stoica et al., 2018).

In this review, we will briefly discuss the pathways responsible for tau degradation, release, and uptake in neurons and glial cells. We will also summarize the potential therapeutic targets and those that are already in clinical trials for promoting tau degradation and preventing tau propagation.

\section{STRUCTURE AND FUNCTION OF TAU IN TAUOPATHIES}

Tau is a microtubule-associated protein encoded by the MAPT gene. Alternative splicing of the single MAPT mRNA generates six different isoforms (Himmler et al., 1989). The longest tau isoform with two-amino terminal inserts and four microtubulebinding domains (2N4R-Tau) has more than 80 phosphorylation sites (Iqbal et al., 2016).

Under normal conditions, tau is constantly phosphorylated and dephosphorylated to regulate the microtubule assembly in axons. Tau is also involved in facilitating the axonal transport of cellular cargoes on microtubule tracks in neurons by differentially regulating two motor proteins, dynein and kinesin (Dixit et al., 2008). In healthy neurons, lower tau concentration in the soma enables kinesin's binding to microtubules and anterograde transport of cargoes to the distal axon. Cargoes are then released due to the higher tau concentration at the distal axon. Whereas dynein-dependent retrograde transport from the axon to soma is not affected because of dynein's lower sensitivity to tau. However, tau-dependent axonal transport is still controversial since it is not validated in in vivo studies. One study even shows tau overexpression or knockout in a mouse model does not show impairment in the axonal transport (Yuan et al., 2008).

Under pathological conditions, tau undergoes various types of post-translational modifications, including hyperphosphorylation and detaches from microtubules. Detached tau can either exist as soluble monomers or form 
neurotoxic oligomeric aggregates, paired helical filaments (PHFs), and ultimately neurofibrillary tangles (NFTs). Loss-offunction of tau, which causes disassembly of the microtubule, and gain-of-function of neurotoxic tau aggregates, have both been proposed during the disease progression of $\mathrm{AD}$ and related tauopathies (Rapoport et al., 2002; Trojanowski and Lee, 2005; Avila et al., 2010).

Tauopathies are a group of more than twenty diseases, where tau is hyperphosphorylated and aggregated to form intracellular inclusions NFTs inside neurons or glial cells (Götz et al., 2019). The most common forms of primary tauopathies are Pick's disease (PiD), Progressive Supranuclear Palsy (PSP), Corticobasal Degeneration (CBD), and frontotemporal dementia with parkinsonism liked to chromosome 17 (FTDP-17). The secondary tauopathy, Alzheimer's disease (AD), is the most prevalent form of tauopathy, affecting more than 50 million people worldwide and 50 million expected to increase to 152 million by 2050 (World Alzheimer Report, 2019). Familial tauopathies are caused by pointmutations in the MAPT gene or alternative splicing of $M A P T$ mRNA - resulting in the imbalance in different tau isoforms, and/or numerous types of post-translational modifications. Accordingly, patients with tauopathies display different brain regional susceptibility and present with distinct clinical symptoms, which is driven by the underlying type and extent of tau pathologies. The molecular pathogenesis of tauopathies is extensively reviewed in another review (Götz et al., 2019).

\section{CLEARANCE OF PATHOLOGICAL TAU BY AUTOPHAGY-ENDOLYSOSOMAL NETWORK}

The ubiquitin-proteasome system (UPS), Chaperon-mediated Autophagy (CMA), and endosomal microautophagy (e-MI) clear soluble neurotoxic tau, while macroautophagy degrades intracellular insoluble tau inside neurons. Additionally, autophagy-independent endolysosomal degradation of ubiquitinated tau with certain phosphorylation sites has also been reported (Figure 1). Tau degradation by UPS is extensively reviewed elsewhere (Lee et al., 2013). Numerous studies have reported that all three different types of autophagy process - CMA (Wang et al., 2009), e-MI, and macroautophagy, participate in the endolysosomal degradation of pathological tau. In brains of $\mathrm{AD}$ subjects, Alz50-positive conformationally altered tau-containing intraneuronal granules exhibited lysosomelike structures in regions surrounding infarcted foci in the human cerebral cortex, suggesting a failure of lysosomal

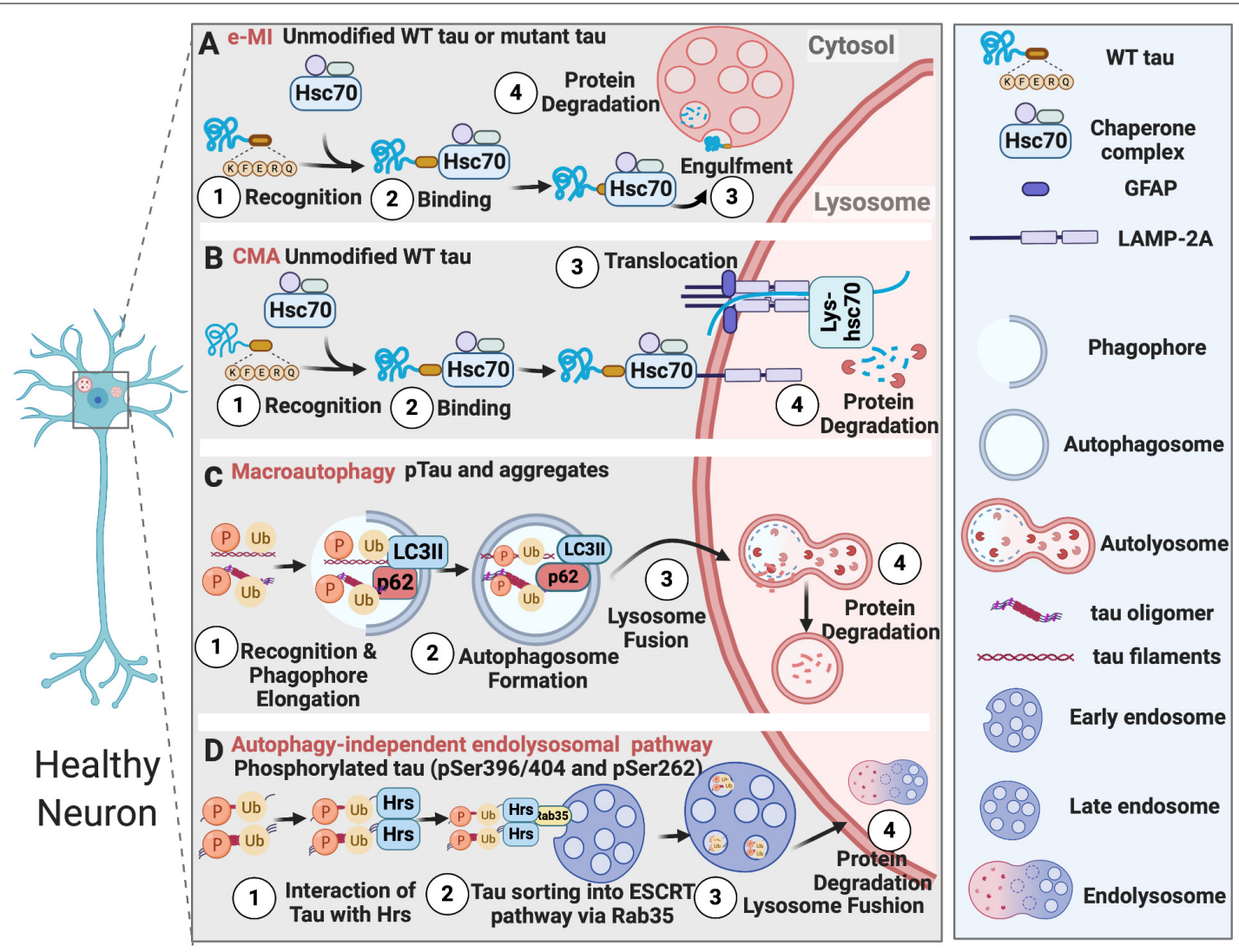

FIGURE 1 | Tau degradation in healthy neurons. (A) Unmodified wildtype tau or mutant tau is degraded by e-MI; (B) Unmodified wildtype tau is degraded by CMA; (C) Phosphorylated tau and tau aggregates are degraded by macroautophagy; (D) Tau phosphorylated at specific sites is degraded by autophagy-independent endolysosomal pathway. 
degradation in tau tangles (Ikeda et al., 1998, 2000). Aside from $\mathrm{AD} /$ tauopathies, the fact that NFTs are also found in brains of subjects with Niemann-Pick type C (NPC), a lysosomal storage disease resulting from lysosomal function defects, suggests endolysosomal failure might be inducing tau aggregation and tangle build-up in the brain (Wang and Mandelkow, 2012).

\section{CMA-Dependent Degradation of Pathological Tau}

CMA is one of the first discovered processes, which can selectively degrade cytosolic components by lysosomes (Dice, 1982). It does not utilize any membrane structures to engulf cargo, but instead utilizes chaperone proteins, such as HSC70, HSP90, HSP40, to recognize cargo proteins that have a KFERQ motif. Then these cargoes are unfolded and translocated directly across the lysosomal membrane for degradation (Cuervo and Wong, 2014; Kaushik and Cuervo, 2018).

During physiological conditions (upon damage or partial unfolding), CMA regulates various biological activities, including DNA repair, glucose/lipid metabolism, cellular reprogramming, and response to stress, through mediating the turnover of cellular non-aggregated proteins that have KFERQ motifs. CMA failure can result in several neurodegenerative diseases, including Parkinson's disease, AD, frontotemporal lobar degeneration (FTLD), amyotrophic lateral sclerosis, and Huntington's disease. Neurodegeneration is often accompanied by the accumulation of CMA substrates like $\alpha$-synuclein, carboxyl-terminal hydrolase isozyme L1 (UCHL1), Tau, and TAR DNA-binding protein 43 (TDP-43) (Cuervo and Wong, 2014; Kaushik and Cuervo, 2018).

CMA-dependent degradation of unmodified wild-type tau was first described by Wang et al. (2009). However, in an inducible mouse neuroblastoma N2a cell model of tauopathy expressing the repeat domain of Tau with an FTDP-17 mutation $\left(\mathrm{Tau}_{\mathrm{RD}} \Delta \mathrm{K} 280\right), \mathrm{Tau}_{\mathrm{RD}} \Delta \mathrm{K} 280$ can only be partially cleaved by a thrombin-like activity in the cytosol to generate the F1 fragment. F1 fragment has two CMA targeting motifs $\left({ }^{336} \mathrm{QVEVK}^{340}\right.$ and $\left.{ }^{347} \mathrm{KDRVQ}^{351}\right)$ and can be targeted to the CMA receptor by the chaperone heat-shock cognate protein 70 (Hsc70) and bind to the lysosomal membrane via lysosomal associated membrane protein 2A (LAMP-2A). However, F1 fragment can only partially translocate into lysosomes. This promotes Cathepsin L-mediated cleavage of F1 fragment at the $\mathrm{C}$ terminus to generate F2 and F3 fragments and further induces $\operatorname{Tau}_{\mathrm{RD}} \Delta \mathrm{K} 280$ oligomerization and aggregation, which can be degraded through the autophagy pathway. The oligomers of $\mathrm{Tau}_{\mathrm{RD}} \Delta \mathrm{K} 280$ can further interfere with lysosomal function and lead to lysosome leakage (Wang et al., 2009).

\section{Macroautophagy-Dependent Degradation of Pathological Tau}

Macroautophagy is a more complex and selective process than CMA. For the macroautophagy to occur, a doublemembrane structure called autophagosome forms around protein aggregates, damaged organelles, and microbes prior to their engulfment. Macroautophagy substrates are recognized by selective autophagy receptors (SAR), such as p62/SQSTM1 (sequestosome-1), which binds to the mammalian ATG8 proteins anchored to the inner membrane of the autophagosome, through LC3-interacting region (LIR) motifs (Johansen and Lamark, 2020). Macroautophagy-dependent degradation of pathological tau and its failure in tauopathies have been reported in numerous studies (Wang and Mandelkow, 2012; Menzies et al., 2017; Lee et al., 2013).

It has been observed that hyperphosphorylated tau colocalizes with LC3-positive vesicles and p62 receptor protein in the post-mortem brains of familial AD, CBD, and PSP patients (Piras et al., 2016). Furthermore, in cultured primary neurons, macroautophagy, but not proteasome, was responsible for the degradation of endogenous tau (Krüger et al., 2012). Aligned with this observation, autophagy inhibition by chloroquine enhanced tau aggregation (Hamano et al., 2008), and autophagy induction with rapamycin reduced tau levels in vitro and in vivo in a Drosophila model expressing human tau. In Parkin-knockout and tau-overexpressing mice, trehalose, which stimulates autophagy, alleviated tau pathology (Rodríguez-Navarro et al., 2010). Pharmacological inhibition of phospholipase D1 (PLD1), which is associated with the endosomal system and located on the outer membrane of the autophagosome, by a small molecule 5-fluoro-2-indolyl des-chlorohalopemide (FIPI) in organotypic brain slices led to an accumulation of Tau and p62 aggregates (Dall'Armi et al., 2010). In summary, these studies suggest the involvement of macroautophagy in the degradation of tau aggregates.

\section{e-MI-Dependent Degradation of Pathological Tau}

Tau is also degraded by another form of autophagy called microautophagy. Microautophagy was considered to be a nonselective autophagic process, which can internalize soluble cytosolic cargoes through invaginations of the lysosomal membrane. However, a selective form of microautophagy, which recognizes cargoes harboring KFERQ motifs with the help of Hsc70 and delivers them into late endosomes, was discovered and named endosomal microautophagy (e-MI) (Sahu et al., 2011). It has been shown that more than half of the synaptic proteins have KFERQ motifs and can be recognized by the chaperone protein Hsc70. They are delivered to late endosomes/multivesicular bodies (MVBs) and degraded in the Drosophila neurons. Therefore, e-MI regulates the turnover of old and damaged synaptic proteins and neurotransmitter release (Uytterhoeven et al., 2015). Interestingly, tau also has two KFERQ motifs, and inducing microautophagy reduced mutant tau levels and synaptic vesicle sequestration at the pre-synaptic terminal in the Drosophila neurons (Uytterhoeven et al., 2018).

As summarized above, all three forms of autophagy can mediate the degradation of tau. However, how these pathways differ or overlap is largely unknown. Caballero et al. (2018) studied two different tau mutations, P301L, which causes autosomal-dominant FTDP-17 (Hutton et al., 1998), and A152T that does not cause autosomal-dominant disease but associates with a higher risk of FTD and AD (Coppola et al., 2012). Interestingly, the degradation of these mutant forms of tau by 
three different forms of autophagy varied in N2a cells. A152T mutation disrupted tau degradation in late endosome by e-MI, while macroautophagy was able to effectively degrade A152T tau, and its degradation by CMA was only mildly affected. Whereas the upregulation of macroautophagy or CMA did not increase the degradation of P301L-tau or reduce its cytotoxicity, suggesting merely upregulating macroautophagy or CMA may not achieve the therapeutic goal in efficiently clearing autosomal-dominant forms of mutated tau. Nonetheless, CMA may be effective in clearing the non-autosomal-dominant type of tau relevant to FTD and AD. On the other hand, e-MI was only able to degrade wild-type tau, but not the mutant forms of tau, a concept needing further evaluation as a recent study (Uytterhoeven et al., 2018) contradicted this conclusion. These findings suggest different tau mutants may favor certain degradative pathways. The differential degradation of tau mutants should be taken into consideration when modulating autophagy for therapeutic intervention in diverse types of tauopathies.

\section{Autophagy-Independent Endolysosomal Degradation of Pathological Tau}

Autophagy-independent endolysosomal degradation of tau was also reported recently in primary hippocampal neurons and N2a cells (Vaz-Silva et al., 2018). Intriguingly, tau was found in Hrs/EEA1/Rab5-positive early endosomes, MVB vesicles, LAMP1-positive vesicles, suggesting its trafficking through the entire endolysosomal pathway. Tau interacted with the initial endosomal sorting complex required for transport (ESCRT) protein Hrs, possibly through ubiquitination, since the interaction was increased by deubiquitylating enzyme inhibitors. Tau sorting into the ESCRT pathway was dependent on the small GTPase Rab35. Rab35-dependent degradation of tau was particularly specific to tau phosphorylated at specific sites. For example, pSer396/404 and pSer262, but not pSer202. This finding again suggested the preferential sorting of specifically phosphorylated tau by multiple AELN pathways.

\section{AUTOPHAGY-ENDOLYSOSOMAL NETWORK DEFECTS IN TAUOPATHY}

Accumulation of lysosomes and lysosomal hydrolases and defects in lysosomal membrane integrity has been reported in $\mathrm{AD}$ patients (Perez et al., 2015). Increased levels of lysosomal components cathepsin D and LAMP1 was also found in the brains of CBD and PSP patients (Piras et al., 2016). The buildup of autophagosomes, autophagic vacuoles, MVBs, multilamellar bodies, and cathepsin-containing autolysosomes is also apparent in the dystrophic neurites in the brains of $\mathrm{AD}$ subjects (Nixon et al., 2005; Nixon, 2013; Menzies et al., 2017), which indicates defects in autophagosome maturation, transport, and fusion with lysosomes during the course of pathological progression (Figure 2).

In healthy neurons, autophagosomes are initially produced at the terminal of axons and transported retrogradely to the soma, where lysosomes are located. This retrograde transport of autophagosomes along the microtubule is dynein- and activity-dependent in granule cells (Katsumata et al., 2010). It was recently reported that soma-derived degradative lysosomes are anterogradely transported to the distal axon to fuse with autophagosomes to form autolysosomes, which are later retrogradely transported to the soma for degradation in mouse cortical neurons at DIV 14 (Farfel-Becker et al., 2019). In disease neurons, impaired axonal delivery of lysosome causes autophagic stress. Whether it contributes to tau pathology needs further investigation.

Additionally, lysosomal $\mathrm{pH}$ dysregulation and aberrant activity of a proton pump-the vacuolar ATPase (v-ATPase) have been implicated in various neurodegenerative diseases, including AD (Colacurcio and Nixon, 2016). Particularly, a brain proteomic analysis (Chang et al., 2013) revealed the expression of v-ATPase H+ Transporting V1 Subunit E1 (ATP6V1E1), which is responsible for the acidification of various intracellular organelles (endosomes, lysosomes, the trans-Golgi network, and synaptic vesicles), is altered throughout the course of tau tangle pathologies. This study utilized a transgenic mouse model (NSEhtau12) expressing human wild-type tau under the control of neuron-specific enolase (NSE). ATP6V1E1's expression was increased in the early disease process, but decreased later, suggesting lysosomal function was augmented upon protein aggregate challenge, then overwhelmed and compromised later as the disease progresses.

\section{GENETIC RISKS ASSOCIATED WITH THE AUTOPHAGY-ENDOLYSOSOMAL SYSTEM IN TAUOPATHIES}

Genome-wide association studies have identified 27 AD susceptibility loci. Many of them (for example, BIN1, PICALM, CD2AP, RIN3, SORL1, GRN, and PLD3) affect AELN functions (Van Acker et al., 2019). Particularly, BIN1, PICALM, and GRN have been implicated in tauopathies.

\section{BIN1}

Bridging Integrator 1 (BIN1) is a cytoplasmic protein involved in vesicle-mediated transport. It is localized on early and late endosomes, lysosomes, and recycling endosomes and can bind to tau, dynamin, and clathrin. In AD brains, neuronal isoform 1 of BIN1 protein level is decreased, and microglial isoform 9 is increased (Holler et al., 2014). Protein levels of BIN1 have been shown to negatively correlate with tau pathology in an in vitro model of BIN1 knockout rat hippocampal neurons, where accumulated tau disrupts endosomes and propagates to the cytosol to induce more aggregates. The underlying mechanism was BIN1 reducing the endocytic flux through Rab5 deactivation (Calafate et al., 2016).

\section{PICALM}

Phosphatidylinositol Binding Clathrin Assembly Protein (PICALM) is a clathrin-assembly protein that can recruit the adaptor complex 2 (AP2) to clathrin-coated pits to regulate membrane cycling. PICALM is an AD risk gene, and singlenucleotide polymorphism (SNP) in PICALM leads to abnormal 


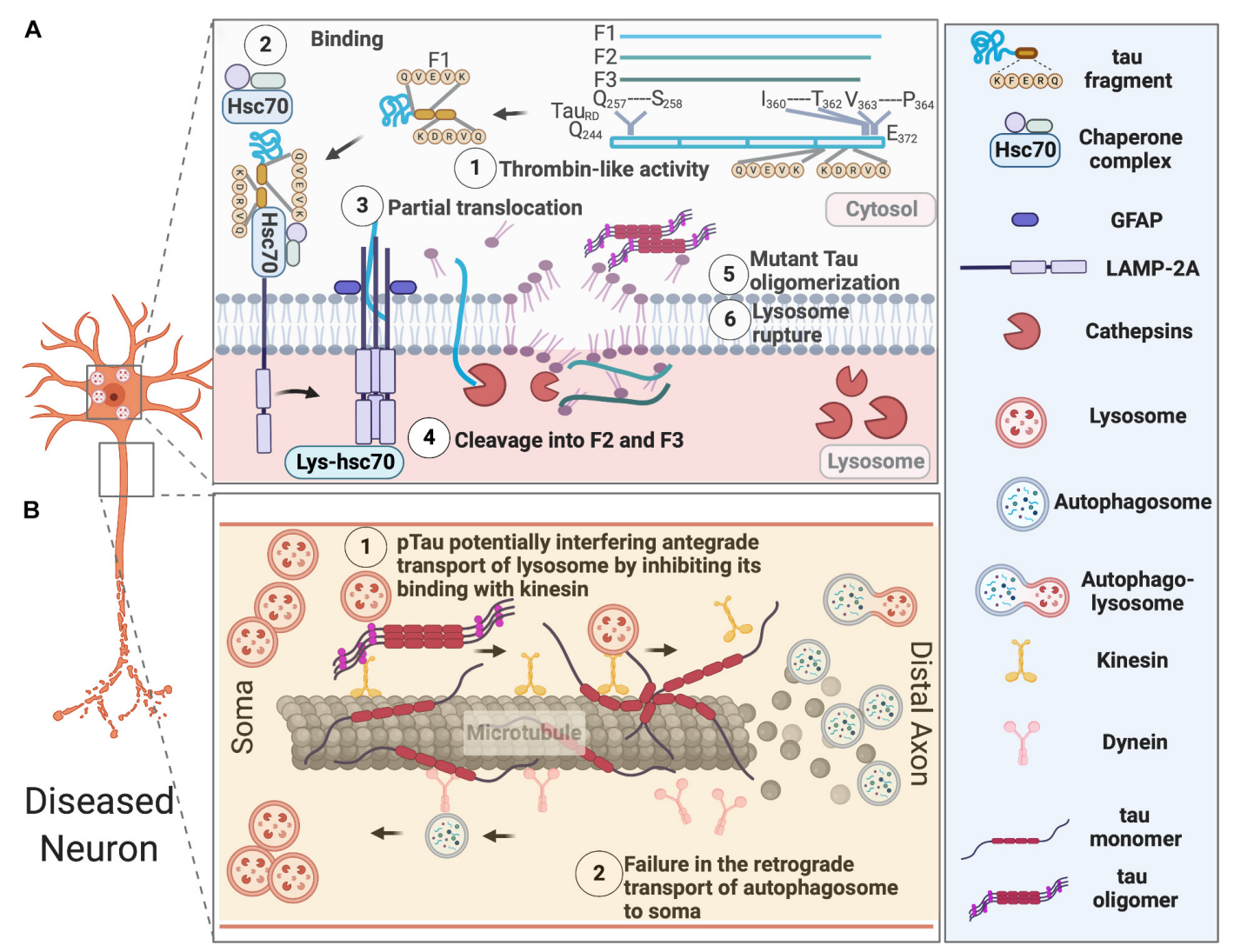

FIGURE 2 | AELN defects in diseased neurons. (A) Exogenously expressed tau repeat domain can be cleaved by a Thrombin-like activity to generate an F1 fragment, which is then recognized by Hsc70 and co-chaperons. Hsc-70 bound F1 fragment can only partially translocate into lysosomes, which further induce its cleavage by Cathepsin L to generate F2 and F3 fragments. Tau fragments impair CMA and cause lysosomal rupture, which in turn induce mutant tau oligomerization and aggregation. (B) Pathological tau undergoes hyperphosphorylation and aggregates in the somatodendritic compartments, overwhelming AELN and also inhibits kinesin's binding to the microtubules, which further interfere with the degradative lysosome transportation to axons. At the later stage, dissociation of hyperphosphorylated tau from microtubule and its destabilization/disassembly may interfere with the retrograde transport of autophagosomes to lysosomes and ultimately lead to accumulation of autophagosomes in axons.

cleavage of PICALM by calpain and caspase-3 (Ando et al., 2013). It has been shown that PICALM modulates autophagy and its depletion leads to autophagic dysfunction, which contributed to the accumulation of phosphorylated tau in primary mouse neurons (Moreau et al., 2014). PICALM colocalizes with phosphorylated $3 \mathrm{R}$ and $4 \mathrm{R}$ tau in the brains of $\mathrm{AD}, \mathrm{FTLD}, \mathrm{PiD}$, and PSP patients (Ando et al., 2016).

\section{GRN}

The mutation of Granulin precursor $(G R N)$, another risk gene for FTD besides MAPT, is also implicated in AD cohorts (Perry et al., 2013). Progranulin (PGRN), encoded by the human GRN gene, is a secreted growth factor that can localize to intraneuronal membrane compartments, including lysosomes. Homozygous GRN mutations can cause a rare lysosomal storage disease (Dickson et al., 2010; Paushter et al., 2018), suggesting a plausible interaction between PGRN and tau in contributing to FTDs.

In addition to the genes mentioned above, mutations in charged multivesicular body protein 2B (CHMP2B) (Skibinski et al., 2005), in the ESCRT-III complex, and C9ORF72, which colocalizes with RAB proteins, are linked to defects in endosomal trafficking in FTD (Farg et al., 2014).

\section{TAU TRANSMISSION}

Mounting evidence now suggests tau, together with many other proteins, involved in neurodegeneration, can propagate like prion from cell to cell either via direct physical synaptic connections or through extracellular vesicles, like exosomes and ectosomes, or by tunneling nanotubules, other endocytic pathways (extensively reviewed in Fuster-Matanzo et al., 2018; Gibbons et al., 2019; Uemura et al., 2020). Tau not only propagates between neurons, but it can also spread from neurons to microglia, astrocytes, and oligodendrocytes (Figure 3). It is speculated that tau might even spread from glial cells to glial cells in tauopathies like PSP and CBD (Narasimhan et al., 2017), eventually forming astrocytic plaques and/or tufted astrocytes.

Spreading of tau pathology to synaptically connected brain regions was initially suggested from the hierarchical pattern of tau pathology beginning in the trans-entorhinal cortex 


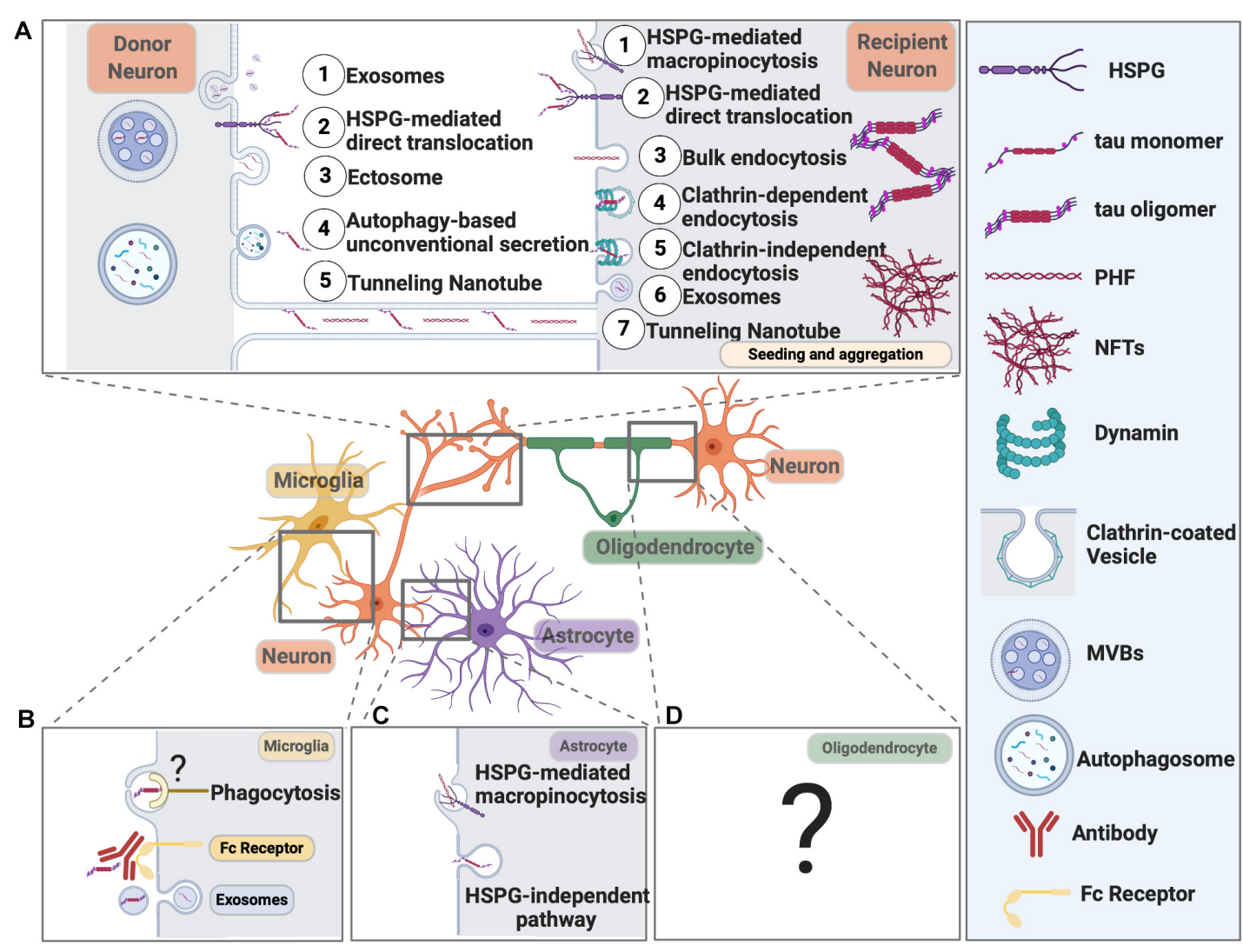

FIGURE 3 | Pathways Mediating Pathological Tau Release and Uptake in Neurons and Glial Cells. (A) Neurons release pathological tau through the following pathways: (1) Exosomes; (2) HSPG-mediated direct translocation; (3) Ectosome; (4) Autophagy-based unconventional secretion; (5) Tunneling nanotube. Neurons can internalize pathological tau through the following pathways: (1) HSPG-mediated macropinocytosis; (2) HSPG-mediated direct translocation; (3) Bulk endocytosis; (4) Clathrin-dependent endocytosis; (5) Clathrin-independent endocytosis; (6) Exosomes; (7) Tunneling Nanotube. (B) Microglial cells internalize tau through exosome and phagocytosis. Microglia can also take up antibody-bound tau fibrils. Internalized tau can be degraded or packaged into exosomes and propagate. The receptor on the microglial cell surface that recognizes pathological tau is yet to be defined. (C) Astrocytes take up tau fibrils through HSPG-mediated macropinocytosis and tau monomers through an HSPG-independent pathway that needs further investigation. (D) The molecular pathways responsible for tau uptake in oligodendrocytes remain elusive.

and eventually spreading to synaptically connected brain regions, such as the hippocampus and, later, the cortex (Braak and Braak, 1995). An in vitro study first showed that extracellular recombinant tau aggregates, but not monomers, were internalized by a mouse immortalized neural progenitor cell line C17.2 and induced fibrillization of intracellular fulllength tau, which was able to further cross-seed fibrillization of recombinant tau monomer (Frost et al., 2009). Another in vitro study (Guo and Lee, 2011) reported the introduction of a small amount of misfolded preformed fibrils of tau (pffs) into QBI293 immortalized human embryonic kidney cells transfected with human tau, turned a large amount of soluble Tau into NFT-like filamentous inclusions. Uptake of pffs into the cells was mediated by endocytosis, and the P301L mutation of tau enhanced pff-induced aggregation accompanied by reduced microtubule stability. Conclusions from these findings were also supported by multiple in vivo studies utilizing pathological tau from different resources in different mouse models.

Injection of brain extract from P301S human tau mice into the brains of ALZ17 transgenic mice expressing wild-type human tau induced seeding of wild-type human tau to become tau filaments and spreading of tau pathology from the injection site to the neighboring brain regions (Clavaguera et al., 2009). Mislocalization of tau from axons to somatodendritic compartments and spreading of tau pathology from the entorhinal cortex to the surrounding brain regions, including the subiculum, CA1, and dentate gyrus was also observed in an inducible mouse line Neuropsin-tTA-Tau (NT), which differentially express tau in the EC region (Liu et al., 2012) and mimics the Braak staging. Tau propagation is dependent on synaptic circuitry since in rTgTauEC mice, a transgenic mouse model exclusively expressing human $\mathrm{P} 301 \mathrm{~L}$ tau in the layer II of the entorhinal cortex (EC), tau pathology initiated from EC transgene-expressing neurons to non-transgene expressing neurons downstream in the synaptic circuit such as the dentate gyrus, hippocampus CA region, and cingulate cortex (De Calignon et al., 2012). Tau propagation theory was further supported by the evidence that synthetic tau pffs alone can seed endogenous tau to become NFTlike structures in a dose- and time-dependent manner in the interconnected brain regions of PS19 mice (Iba et al., 2013). 


\section{Tau Release From Neurons}

For tau to spread between cells, it first needs to be released from neurons. Studies have shown that in addition to being passively released from dead cells, tau can also be actively released from neurons in vesicle-associated form or non-vesicle-associated free form (Tables 1, 2).

Exosomes are extracellular vesicles, which are formed inside an MVB. Exosome release into the extracellular space is dependent on the fusion of the MVB with the plasma membrane. Secreted tau in the supernatant of M1C neuroblastoma cells was exosome-associated and mostly enriched for pT181 (AT270

TABLE 1 | Pathways mediating physiological tau release from neurons.

\begin{tabular}{|c|c|c|c|}
\hline Pathways & Forms of Tau & Characteristics & References \\
\hline $\begin{array}{l}\text { Non-vesicular } \\
\text { secretion }\end{array}$ & Free form & $\begin{array}{l}\text { Majority of Secreted tau is in } \\
\text { free, non-vesicular form. } \\
\text { Whether it is HSPG- } \\
\text { dependent was not examined. }\end{array}$ & $\begin{array}{l}\text { Dujardin et al., } \\
2014\end{array}$ \\
\hline $\begin{array}{l}\text { Non-exosome } \\
\text { associated }\end{array}$ & $\begin{array}{l}\text { Physiological tau, } \\
\text { non-aggregating } \\
\text { form }\end{array}$ & $\begin{array}{l}\text { AMPA-mediated, } \\
\text { calcium-dependent tau } \\
\text { release enhanced with } \\
\text { increased neuronal activity. } \\
\text { Not clear if this pathway } \\
\text { involves other vesicles. }\end{array}$ & $\begin{array}{l}\text { Pooler et al., } \\
2013\end{array}$ \\
\hline Ectosome & $\begin{array}{l}\text { Dephosphorylated, } \\
\text { full-length tau or } \\
\text { C-terminus } \\
\text { truncated }\end{array}$ & $\begin{array}{l}\text { Found in mouse ISF samples, } \\
\text { slightly switches to exosomal } \\
\text { secretion when tau } \\
\text { accumulates inside cells. }\end{array}$ & $\begin{array}{l}\text { Dujardin et al., } \\
\text { 2014; Cocucci } \\
\text { and Meldolesi, } \\
2015\end{array}$ \\
\hline $\begin{array}{l}\text { Autophagy- } \\
\text { based } \\
\text { unconventional } \\
\text { secretion }\end{array}$ & Total tau & $\begin{array}{l}\text { Released into media without } \\
\text { any stimulation in SHSY-5Y } \\
\text { cells }\end{array}$ & $\begin{array}{l}\text { Kang et al., } \\
2019\end{array}$ \\
\hline
\end{tabular}

TABLE 2 | Pathways mediating pathological tau release from neurons.

\begin{tabular}{|c|c|c|c|}
\hline Pathways & Forms of tau & Characteristics & References \\
\hline Exosome & $\begin{array}{l}\text { Total tau, pT181- } \\
\text { tau, pS396-tau, } \\
\text { fragmented tau } \\
\text { lacking C-Terminus }\end{array}$ & $\begin{array}{l}\text { Neuronal activity- and } \\
\text { synaptic connectivity- } \\
\text { dependent }\end{array}$ & $\begin{array}{l}\text { Saman et al., } \\
\text { 2011; Dujardin } \\
\text { et al., 2014; Wang } \\
\text { et al., } 2017\end{array}$ \\
\hline Ectosome & $\begin{array}{l}\text { Fragmented tau } \\
\text { lacking C-Terminus }\end{array}$ & $\begin{array}{l}\text { Slightly switches to } \\
\text { exosomal secretion } \\
\text { when tau accumulates } \\
\text { inside cells. }\end{array}$ & $\begin{array}{l}\text { Dujardin et al., } \\
2014\end{array}$ \\
\hline $\begin{array}{l}\text { Tunneling } \\
\text { Nanotube }\end{array}$ & $\begin{array}{l}\text { Exogenous tau } \\
\text { monomers and } \\
\text { fibrils }\end{array}$ & $\begin{array}{l}\text { Filamentous-actin- } \\
\text { containing } \\
\text { membranous } \\
\text { structures }\end{array}$ & $\begin{array}{l}\text { Tardivel et al., } \\
2016\end{array}$ \\
\hline $\begin{array}{l}\text { HSPG- } \\
\text { mediated direct } \\
\text { translocation }\end{array}$ & $\begin{array}{l}\text { Soluble full-length } \\
\text { tau, phosphorylated, } \\
\text { oligomeric }\end{array}$ & $\begin{array}{l}\text { Secreted soluble tau } \\
\text { utilized the same } \\
\text { HSPG-mediated direct } \\
\text { translocation } \\
\text { mechanism to } \\
\text { transcellularly enter the } \\
\text { recipient cells and } \\
\text { induced aggregation }\end{array}$ & $\begin{array}{l}\text { Katsinelos et al., } \\
\text { 2018; Merezhko } \\
\text { et al., } 2018\end{array}$ \\
\hline $\begin{array}{l}\text { Autophagy- } \\
\text { based } \\
\text { unconventional } \\
\text { secretion }\end{array}$ & $\begin{array}{l}\text { Various forms of } \\
\text { pTau }\end{array}$ & Nigericin induced & Kang et al., 2019 \\
\hline
\end{tabular}

antibody site), an established early AD biomarker used in CSFbased AD diagnosis. The authors also found that CSF samples from mild $\mathrm{AD}$ patients had an increased level of exosomeassociated pT181 tau (Saman et al., 2011). The exosome-mediated release and trans-synaptic transmission of Tau between neurons are dependent on neuronal activity and synaptic connectivity, as shown by an in vitro microfluidic study (Wang et al., 2017). Interestingly, exosomal tau was taken up by neurons and microglia, but not astrocytes, and microglia was more efficient in internalizing exosomal tau. Exosomal tau released from N2a cells or monomeric or oligomeric tau from exosomes derived from $\mathrm{AD}$ patients CSF samples can both induce tau aggregation in cultured cells.

Besides exosomes, another type of vesicles, ectosomes (Cocucci and Meldolesi, 2015), is also shown to be involved in tau secretion. Ectosomes vesicles $(0.1-1 \mu \mathrm{m}$ in diameter) that are budded and released directly from the plasma membrane upon elevated levels of intracellular calcium, inflammatory molecules, or oxidative stress. One study showed that in addition to the major non-vesicular free form of tau is predominantly secreted in ectosomes in vitro and in vivo under physiological conditions (Dujardin et al., 2014). Ectosomal tau was also found in the interstitial fluid (ISF) of mice in the same study. This physiologically secreted tau is mainly not phosphorylated. However, intracellular pathological accumulation of tau leads to a slight shift toward tau secretion by exosomes (Dujardin et al., 2014). Interestingly, exo- and ecto-somal tau appears to be lacking the C-terminus, indicating tau truncation (GuillozetBongaarts et al., 2007), which is another sign of tau pathology. Some important questions remain to be elucidated: (1) whether pathological tau secreted via ectosomes can be internalized and induce tau seeding in neurons; (2) whether ectosomal tau can be detectable in biological fluids (ISF, CSF, or blood) of tauopathy patients; (3) what are the molecular pathway(s) responsible for ectosomal tau release.

The third mechanism, Heparan sulfate proteoglycan (HSPG)mediated direct translocation across the plasma membrane, was responsible for the secretion of soluble full-length tau in vitro in $\mathrm{CHO}$ cells with monomeric tau binding to $\mathrm{PI}(4,5) \mathrm{P} 2$ containing liposomes, which resemble the inner leaflet of the plasma membrane (Katsinelos et al., 2018). The secreted soluble tau utilized the same HSPG-mediated direct translocation mechanism to transcellularly enter the recipient cells and induced aggregation of the GFP-tagged tau repeat domain (RD-GFP). Another study reported when overexpressing 0N4R isoform of tau in N2a cells led to the clustering of phosphorylated, oligomeric tau at the plasma membrane and then penetrate the plasma membrane to be secreted to the extracellular space, partially mediated by HSPG (Merezhko et al., 2018).

Exogenous tau monomers and fibrils are also reported to increase the number of tunneling nanotubes (TNTs), filamentous-actin-containing membranous structures that bridge and connect cells, which can facilitate the intercellular transfer of tau between neurons (Tardivel et al., 2016). However, the exact molecular mechanism of the TNT formation remains elusive, and the relevance of this occurring in vivo needs further investigation. 
Interestingly, stimulating neuronal activity or inducing the $\alpha$-amino-3-hydroxy-5-methyl-4-isoxazolepropionic acid receptor (AMPA receptor) with its agonist $(S)$-AMPA prompted tau release from healthy, mature primary cortical neurons (Pooler et al., 2013). In contrast, suppressing presynaptic vesicle release by tetanus toxin or inhibiting neuronal activity with tetrodotoxin both significantly blocked AMPA-mediated, calcium-dependent tau release. However, this mode of tau secretion was not associated with exosome, suggesting physiological tau release might differ from the unconventional secretion of pathological extracellular tau. This observation was confirmed by a subsequent in vivo study where the authors used in vivo micro-dialysis and found neuronal activity rapidly increases the preexisting tau in brain interstitial fluid (ISF) within hours in a transgenic mouse expressing a non-aggregating form of human tau (Yamada et al., 2014). However, the clearance of ISF tau was not rapid and suggested to take a longer time, maybe several days. Notably, this study did not examine other pathways involved in tau secretion, for example, HSPG-mediated direct translocation, ectosomes, and TNTs. Therefore, the involvement of other tau secretory pathways for clearing ISF-tau cannot be completely ruled out.

Similar to physiological tau release, pathological tau release was also enhanced with increased neuronal activity both in vitro and in vivo. Endogenous full-length tau derived from donor neurons from rTg4510 mice - expressing human P301L tau, was released into the extracellular media (although vesicle-associated or not is unclear) and taken up by recipient neurons to form tau aggregates. This pathological tau further induced neurofibrillary tangle formation in distant cells (Wu et al., 2016). Neuronal activity induction by an optogenetic approach increased tau release and cell-to-cell propagation in vitro. Optogenetically inducing neuronal activity also enhanced tau pathology and hippocampal cell layer atrophy in the stimulated hemisphere compared to the unstimulated hemisphere of $\mathrm{rTg} 4510$ mice. This finding was confirmed by using a chemogenetic approach to induce neuronal activity in the EC of a separate mouse line called "EC-tau." Besides, tau released from human iPSC-derived neurons was also able to transfer to recipient donor neurons through the extracellular space. Although non-mutant tau, either from wildtype mice or from non-mutant human iPSCs, can also be released and internalized. Yet, this tau did not form neurofibrillary tangles even with prolonged time of culturing, suggesting the phosphorylated, aggregated tau or mutant tau can more easily accumulate and seed in the recipients rather than the soluble, non-mutant form of tau.

Apart from the degradation of intracellular components, autophagy has also been shown to regulate the ER- and Golgiindependent unconventional secretion of several leaderless proteins, like cystic fibrosis transmembrane conductance regulator (CFTR) (Gee et al., 2011), IL-1 $\beta$ (Dupont et al., 2011), and High Mobility Group Box 1 (HMGB1) (Thorburn et al., 2009). A recent study (Kang et al., 2019) suggested autophagybased unconventional secretion of tau. Authors treated SHSY-5Y human neuroblastoma cells with bacterial toxin nigericin, which is also a potassium ionophore that can accumulate autophagic vesicles by raising the $\mathrm{pH}$ of acidic cellular compartments. They found both total tau and various types of phosphorylated tau are secreted into the supernatant, which can be inhibited through autophagic protein beclin1 knockdown. This tau secretion process took place without cell death. However, important questions still exist. For example, (1) whether Golgi reassembly-stacking protein of $55 \mathrm{kDa}$ (GRASP55), an important protein involved in autophagy-based unconventional secretion of mammalian leaderless proteins, is involved in the autophagybased tau secretion; (2) whether autophagy proteins are present in the same supernatant; and (3) whether tau is secreted in the same way in human primary microglia and in vivo.

\section{TAU UPTAKE BY NEURONS, MICROGLIA, AND ASTROCYTES}

Secreted Tau can be internalized by recipient neurons, microglia, astrocytes, and oligodendrocytes via various mechanisms, including HSPG-mediated direct translocation, HSPGmediated endocytosis, exosome-based uptake, tunneling nanotube, clathrin-independent endocytosis, clathrin-dependent endocytosis, and HSPG-dependent macropinocytosis.

\section{Uptake Into Neurons}

Most studies have been focused on the uptake of tau into neurons. Neurons can internalize tau through HSPG-mediated direct translocation, exosomes, bulk endocytosis, tunneling nanotube, as mentioned above. Additionally, Clathrin-independent endocytosis and HSPG-dependent macropinocytosis are also shown to be involved in the internalization of Tau by neurons (Table 3).

Full-length tau fibril uptake into neurons was macropinocytosis-dependent as HSPG inhibition blocked tau uptake and aggregation both in primary hippocampal neurons and in WT mice (Holmes et al., 2013). Another study has shown that exogenously added recombinant tau can misfold to form low molecular weight (LMW) aggregate, which can further assemble into short fibrils. Both LMW tau aggregates and short fibrils can be taken up by neurons through bulk endocytosis, but not monomers, long fibrils, nor long filaments (Wu et al., 2013). The internalization takes place either at the axon terminals and then tau being retrogradely transported to the cell body or happens at the somatodendritic compartment and then tau being delivered to the distal terminal axons. Internalized tau aggregates co-localize with late endosomes and lysosomes and potentially overload the proteasome and AELN system. It has been shown that exogenous tau can also be internalized via clathrin-dependent endocytosis and rupture endosome to propagate, which is negatively regulated by neuronal isoform 1 of BIN1 by inhibiting Rab5 and endosome formation in vitro (Calafate et al., 2016).

Autophagy is also linked to the degradation of monomeric tau internalized via clathrin-independent endocytosis within neurons, which further prevent tau seeding (Falcon et al., 2018a). To be specific, fluorescently labeled monomeric and assembled recombinant P301S tau was taken up by HEK293T or SH-SY5Y cells expressing P301S 1N4R tau. This uptake was mediated through clathrin-independent endocytosis. Internalized tau 
TABLE 3 | Pathways mediating pathological tau uptake into neurons.

\begin{tabular}{|c|c|c|c|c|}
\hline Pathways & Forms of tau & $\begin{array}{l}\text { Recipient } \\
\text { cells }\end{array}$ & Subsequent activities inside the recipient cell & References \\
\hline Exosome & $\begin{array}{l}\text { Monomeric or oligomeric tau from exosomes } \\
\text { derived from AD patient CSF, pT181 tau }\end{array}$ & Neuron & Seeding and Propagation & $\begin{array}{l}\text { Saman et al., 2011; } \\
\text { Wang et al., } 2017\end{array}$ \\
\hline $\begin{array}{l}\text { Clathrin-independent } \\
\text { endocytosis }\end{array}$ & Monomeric tau & Neuron & $\begin{array}{l}\text { Seeding and inducing aggregates, rupture } \\
\text { endosome to activate Galectin8-NDP52 mediated } \\
\text { autophagic degradation of monomeric tau }\end{array}$ & Falcon et al., 2019 \\
\hline $\begin{array}{l}\text { HSPG-mediated direct } \\
\text { translocation }\end{array}$ & GFP-tagged tau repeat domain & Neuron & Seeding and Propagating & $\begin{array}{l}\text { Katsinelos et al., 2018; } \\
\text { Merezhko et al., } 2018\end{array}$ \\
\hline Bulk endocytosis & LMW tau aggregates and short fibrils & Neuron & Clogging up UPS and AELN & Wu et al., 2013 \\
\hline Tunneling nanotube & Exogenous Tau monomers and fibrils & Neuron & Unknown & Tardivel et al., 2016 \\
\hline $\begin{array}{l}\text { HSPG-dependent } \\
\text { macropinocytosis }\end{array}$ & Full-length tau fibrils & Neuron & Seeding and Propagating & $\begin{array}{l}\text { Holmes et al., 2013; } \\
\text { Puangmalai et al., } 2020\end{array}$ \\
\hline $\begin{array}{l}\text { Clathrin-dependent } \\
\text { endocytosis }\end{array}$ & Exogenous TauP301L-GFP aggregates & Neuron & $\begin{array}{l}\text { Rupture endosome to propagate, which can be } \\
\text { inhibited by neuronal BIN1 isoform }\end{array}$ & Calafate et al., 2016 \\
\hline
\end{tabular}

induced seeded aggregation of tau and further provoked the formation of tau-containing vesicles positive for Rab5 (endosomal marker) and Galectin-8, suggesting tau aggregates ruptured endosome, which can be detected by Galectin- 8 . Monomeric tau seeds were positive for nuclear dot $52 \mathrm{KDa}$ protein (NDP52), and seeded tau aggregates were positive for p62, suggesting autophagy is activated to degrade the monomeric tau seeds and seeded tau aggregates through different autophagic receptors. siRNA knockdown of autophagosome components LC3C (also the specific binding partner of NDP52) and FAK family kinase-interacting protein of $200 \mathrm{kDa}$ (FIP200) further increased seeded tau aggregation. On the other hand, expressing NDP52 lacking galectin-8-binding (NDP52L374A), LC3Cbinding (NDP52V136S), or the SKICH domain (NDP52 $\Delta 1-$ 126) was not able to reduce seeded tau aggregation (Falcon et al., 2018a). This finding indicates that NDP52-dependent autophagic degradation of monomeric tau seeds through galectin- 8 is important in limiting the seeding and propagation of pathological tau.

A previous study showed that aberrant activation of p300/CBP, which acetylates tau, promotes its aggregation and inhibits its degradation by the proteasome, was observed in tauopathies (Min et al., 2010). The following study reported an additional role of p300/CBP in impairing autophagy-lysosomal pathways and increasing tau secretion in neurons. Overexpression of p300/CBP significantly increased the secretion of total tau and AT8-positive phosphorylated tau by inhibiting the autophagy flux in vitro, while inhibition of p300/CBP reduced synthetic tau fibril-induced seeding activity in vitro and in vivo. A new small inhibitor of p300/CBP, which can reduce tau secretion, was also identified through a high throughput screening (Chen et al., 2020). However, this study did not specify how p300/CBP is increasing tau secretion and which secretory pathway it utilizes. These studies emphasize the important roles of macroautophagy in degrading intracellular total tau, phosphorylated tau, internalized monomeric tau, and synthetic tau fibrils and further preventing the secretion and seeding of pathological tau.

In addition to tau uptake into neurons, glial tau pathology is common in various tauopathies. However, how glial cells internalize tau and what happens subsequently during healthy and disease conditions are still unclear. Table $\mathbf{4}$ summarizes the current state of knowledge about tau uptake by glial cells.

\section{Uptake Into Microglia}

As the major immune cells in the brain, microglial cells are important for the maintenance of homeostasis of the brain by clearing toxic substances by phagocytosis, immune surveillance, engulfing synapses, and mediating neurotransmitter turnover. Regarding tau, microglia can engulf tau aggregates bound to the anti-tau antibody, which is an important underlying mechanism of action for the tau passive immunization studies.

However, microglia can be a foe in spreading tau pathology and exacerbating the disease. Activating microglia in the hTau mouse model by knocking out $C \times 3 c r 1$, the microglial fractalkine receptor (CX3CR1) that inhibits microglial activation, significantly increased AT180 tau hyperphosphorylation signal in the neurons from subiculum at 24 months of age, which correlated with CD45-positive microglia. Whereas at 2 months of age, only the CA1 region had AT180-positive neurons and CD45-positive neurons. These data suggest microglia activation might drive the spatiotemporal, anterograde-based spread of tau pathology in the anatomically connected regions of the hippocampus, from CA1 to subiculum (Maphis et al., 2015).

It has been shown that microglia can propagate pathological tau in the exosome-associated form (Asai et al., 2015). Depleting microglia in an AAV-based rapid human tau $\mathrm{P} 301 \mathrm{~L}$ propagation mouse model drastically suppressed tau propagation. Cultured primary mouse microglia were able to efficiently phagocytose exogenously added pre-aggregated human tau oligomers and also secrete exosomes containing ubiquitinated tau. This purified exosomal tau secreted from microglia was fluorescently labeled and stereotactically injected to the brains of wild-type mice. They were found to propagate inside the brain from the injection site to other brain regions, while exosomes with no tau or equal amount of soluble tau did not transmit inside the brain (Asai et al., 2015).

Microglial BIN1 has also been implicated in tau spreading. Unlike neuronal BIN1 isoform 1, which inhibits RAB5 activation and endosome formation to reduce tau propagation inside neurons, microglial BIN1 isoform 9 is shown to promote tau 
TABLE 4 | Pathways mediating pathological tau uptake into glial cells.

\begin{tabular}{|c|c|c|c|c|}
\hline Pathways & Forms of tau & Recipient cells & $\begin{array}{l}\text { Subsequent activities inside the } \\
\text { recipient cell }\end{array}$ & References \\
\hline Exosome & $\begin{array}{l}\text { Pre-aggregated human tau oligomers, } \\
\text { hyperphosphorylated, oligomeric pT181 tau }\end{array}$ & Microglia & $\begin{array}{l}\text { Release through exosomes and seeding, } \\
\text { microglial BIN1 isoform 9-mediated }\end{array}$ & $\begin{array}{l}\text { Asai et al., 2015; Wang et al., } \\
\text { 2017; Crotti et al., } 2019\end{array}$ \\
\hline Phagocytosis & Anti-tau antibody bound aggregated tau & Microglia & Degradation & Luo et al., 2015 \\
\hline $\begin{array}{l}\text { HSPG-dependent } \\
\text { Macropinocytosis }\end{array}$ & Pre-formed tau fibrils & Astrocytes & Degradation by macroautophagy & Martini-Stoica et al., 2018 \\
\hline HSPG-independent pathway & Exogenous tau monomers & Astrocytes & Unknown & Perea et al., 2019 \\
\hline
\end{tabular}

propagation by incorporating tau into exosomes (Crotti et al., 2019). BIN1 and hyperphosphorylated, oligomeric pT181 tau coexisted from the extracellular vesicles (EVs) containing exosomes purified from CSF samples of AD patients. BIN1-associated, taucontaining EVs induced seeding in an in vitro Tau-FRET assay. Overexpression of the ubiquitous BIN1 isoform 9 induced EVassociated tau release in vitro while the exclusively neuronal BIN1 isoform 1 did not. AAV-mediated overexpression of BIN1 isoform 9 in the hippocampus of PS19 mice worsened tau pathology, whereas BIN1 isoform 1 overexpression did not change pTau immunoreactivity. Knocking out microglial Bin1 from mice resulted in a significant reduction of tau in the EVs, suggesting microglial BIN1 is involved in the "packaging" of tau into the EVs. Conditional knockout of microglial Bin1 in PS19 mice significantly reduced tau spreading compared to its Bin1-expressing littermates. These observations suggest that microglial BIN1 isoforms can contribute to the release of EV-associated tau during tau pathology (Crotti et al., 2019). This study provides another evidence that microglial cells can internalize and propagate pathological tau potentially through the exosomal/endosomal system.

\section{Uptake Into Astrocytes}

Even though astroglial tau pathology is commonly seen in various tauopathies, the exact mechanisms of how astrocytes internalize pathological tau and the subsequent cellular processes after internalization are still elusive. Very few studies have investigated astroglial uptake of tau. It has been shown that pffs of tau can be internalized by astroglial cells through HSPG-mediated micropinocytosis (Martini-Stoica et al., 2018) (discussed in detail in the TFEB section below). Exogenously added tau monomers are reported to be taken up by astrocytes through an HSPGindependent pathway yet needs to be defined (Perea et al., 2019).

\section{Uptake Into Oligodendrocytes}

A recent study injected brain homogenates from subjects with sporadic $\mathrm{AD}$, primary age-related tauopathy (PART), pure agingrelated tau astrogliopathy (ARTAG), globular glial tauopathy (GGT), PSP, PiD, and frontotemporal lobar degeneration linked to P301L mutation (FTLD-P301L) into the ipsilateral corpus callosum of adult wildtype mice induced tau seeding of murine tau in the contralateral corpus callosum (Ferrer et al., 2019). Phospho-tau deposits were found mainly in oligodendrocytes. However, the exact molecular mechanism mediating tau uptake into oligodendrocytes was not studied.

\section{Propagation and Seeding Capacity of Different Tau Strains}

Still, the jury is out on whether monomeric, oligomeric, or fibrillar tau is the most toxic form, a lot of research focus has been on studying the propagation and seeding capacity of tau with different forms and different strain composition. Several studies now have shown that the conformation and size of tau aggregates determine its uptake. Short tau filaments seem to have the greatest seeding capacity.

Pathological tau indeed acts like prion that stably expressed tau repeat domain $(\mathrm{RD})$ can propagate in a clonal fashion in cell cultures. The introduction of this tau RD into naïve cells can produce identical clones. Different tau strains can induce distinctive tau pathologies in naïve P301S mice, which can be steadily detected in three generations of tau inoculated P301S mice (Sanders et al., 2014). Of notice, by using the monoclonal Tet Off-tau RD-YFP cell line system, the authors found that tau RD strains derived from the brain homogenates from 29 patients with AD, Argyrophilic grain disease (AGD), PiD, and PSP displayed distinctive strain compositions across the diseases (Sanders et al., 2014). AD strains showed notable homogeneity, while most non-AD patient samples produced two or more strains with varying ability to cross-seed, which suggests the phenotypic diversity in the tauopathies (Sanders et al., 2014).

AD-patient derived tau fibrils also exhibited differential seeding potency and distinct conformational features from the synthetic, overexpressed tau fibrils, as shown in an in vivo seeding and propagation study in non-transgenic mice (Guo et al., 2016). Indeed, cryo-electron microscopy (cryo-EM) studies revealed that the heparin-induced tau polymers are different from those from $\mathrm{AD}$ or $\mathrm{PiD}$, which have larger cores with different repeat compositions (Zhang et al., 2019), suggesting the complexities of tau fibrilization and seeding abilities in different tauopathies.

The differential seeding capacity of different tau strains or synthetic versus human brain-derived tau fibrils to form distinct aggregates and propagate may be related to the ultrastructural polymorphs of tau aggregates in different diseases. Recent cryoEM studies have found that tau fibrils from different tauopathies present unique conformers. While $\mathrm{AD}$ and chronic traumatic encephalopathy (CTE) have distinct tau filaments contain both 3R and 4R tau (Fitzpatrick et al., 2017), tau filaments from PiD consist of residues Lys254-Phe378 of 3R tau (Falcon et al., 2018b) and those from CBD have four-layered fold with a large non-proteinaceous density (Zhang et al., 2020). In vitro ultrasensitive cell-free tau seed amplification assay also 
showed AD brain and CTE brain had seeding capacities that were orders of magnitude higher than those from PiD brains (Kraus et al., 2019). A very recent study examined neuronal internalization of soluble tau aggregates (oligomers) derived from brains of patients with AD, PSP, and Dementia with Lewy bodies (DLB). They found HSPG-mediated micropinocytosis is responsible for tau uptake in $\mathrm{AD}$ and $\mathrm{DLB}$ conditions, while the HSPG-mediated micropinocytosis and other pathways in other tauopathies are yet to be defined (Puangmalai et al., 2020). This study further suggested preferential uptake of different tau strains by different pathways, which further affect seeding and propagation of tau.

\section{AMELIORATING TAU PATHOLOGY THROUGH AELN MODULATION BY TARGETING TFEB, THE MASTER REGULATOR OF AUTOPHAGY AND LYSOSOME BIOGENESIS}

Transcription factor EB (TFEB) is a master activator for the expression of many lysosomal genes (ATP6V1H, ATP6V0E1, ATP6V1E1, and LAMP1) and genes related to lysosome biogenesis and function (CSTB, M6PR, IGF2R2009, and CTSD), and autophagy genes (SQSTM1, MAP1LC3B, UVRAG, and ATG9B) (Sardiello et al., 2009; Settembre et al., 2011; Chauhan et al., 2013). TFEB is expressed in neurons, microglial, and astroglial cells. In human FTD and CBD brains, transcriptional levels of TFEB and LAMP1, protein levels of LAMP1, and cathepsin D (CTSD) increased compared to normal controls (Martini-Stoica et al., 2018). Transcriptional levels of human brain TFEB also positively correlated with cognitive decline in non-demented, mild cognitive impairment, and demented individuals (Martini-Stoica et al., 2018).

Various studies have shown that TFEB induction can reduce tau pathology and improve behavioral and synaptic functions both in vitro and in vivo. Adeno-associated virus (AAV)-TFEB injection into the lateral ventricles of both cerebral hemispheres on postnatal day 0 (P0) of rTg4510 mouse brains reduced neurofibrillary tangle pathology and reversed behavioral and synaptic deficits compared to their wildtype littermate controls when examined at 4-month post-injection time point (Polito et al., 2014). TFEB particularly degraded soluble and aggregated tau through autophagy and lysosomal activity by upregulating autophagy proteins (LC3B and p62) and lysosomal proteins (LAMP1 and CTSD), more specifically targeting phosphatase and tensin homolog (PTEN) in vitro (Polito et al., 2014). Overexpressing TFEB in another mouse model - P301S/flagTFEB double-transgenic mice enhanced autophagy, restored neuronal loss, reduced PHF tau, reversed age-related deposition of lipofuscin granules, and rescued spatial, working, and reference memories (Wang et al., 2016).

High-content screening of FDA-approved drugs library identified two drugs, Flubendazole and Bromhexine. Bromhexine's effect might be attributed to its metabolite ambroxol, which is linked to TFEB activation. Both drugs act as autophagy inducers through activating TFEB's nuclear translocation by disrupting dynamic microtubule that leads to mTOR deactivation and dissociation from lysosomes (Chauhan et al., 2015). Interestingly, both drugs cleared S202 and S396/S404 hyperphosphorylated tau in vitro in $\mathrm{N} 2$ a cells transfected with 0N3R-T231D/S235D phospho-mimicking human tau followed by treatment with conditioned media from RAW macrophages primed with LPS to induce hyperphosphorylation of tau. The clearance of pathological tau by these two drugs was autophagy-dependent, since knocking down Beclin1, a key autophagy protein, resulted in hyperphosphorylated tau accumulation. Considering the low toxicity of Flubendazole, further investigation should be completed in in vivo tauopathy models. Another study (Currais et al., 2014) discovered the pathological tau reducing effect of Fisetin, an organic flavonoid contained in many types of fruits and vegetables, can mitigate learning and memory deficits in APPswe/PS1dE9 double transgenic $\mathrm{AD}$ mice. Fisetin treatment in mouse primary cortical neurons and rat primary cortical neurons reduced S262 and S396/S404 hyperphosphorylated tau, decreased sarkosyl-insoluble tau in 293T cells co-transfected with plasmids expressing tau and constitutively active HA-GSK-3 $\beta$-S9A. Fisetin-mediated pathological tau degradation was autophagydependent since chemical inhibitors of the autophagy-lysosome pathway decreased tau degradation. Its molecular mechanism of action was also identified as fisetin-mediated mammalian target of rapamycin complex 1 (mTORC1) inhibition, which leads to activation of TFEB and nuclear factor erythroid 2related factor 2 (Nrf2), two transcription factors important for autophagy induction (Currais et al., 2014). A novel small molecule, which can activate TFEB, was also identified as Curcumin Analog C1 (C1) (Song et al., 2020). C1 effectively activated TFEB through direct binding, increased autophagylysosome activity, decreased amyloid pre-cursor protein (APP), APP $\mathrm{C}$-terminal fragments $(\mathrm{CTF}-\beta / \alpha), \beta$-amyloid peptides, and tau aggregates respectively in $5 x F A D, P 301 S$, and $3 x T g-$ $\mathrm{AD}$ mice, and also improved synaptic and cognitive function (Song et al., 2020).

The possibility of Opto-therapeutics to treat $\mathrm{AD}$ and other related tauopathies was demonstrated in a new study (Binder et al., 2020) that showed optical stimulation upon blue-light $(465 \mathrm{~nm})$ illumination can lead to TFEB expression, translocation into the nucleus, and activation, which can further induce autophagy and lysosomal gene expression and ultimately clear pathological Tau from neuronal cells and iPSC-differentiated neurons from an AD patient.

Intriguingly, a cell-specific contribution of TFEB in attenuating extracellular tau propagation and upregulating extracellular tau degradation was attributed to astrocytes both in vivo and in vitro (Martini-Stoica et al., 2018). AAV-TFEB overexpression in primary mouse astrocytes not only boosted the expression levels of lysosomal proteins, LAMP1, cathepsin B (CTSB), and CTSD but also increased the uptake of synthetic tau fibrils of the truncated form of human tau with the P301L mutation (K18 P301L). TFEB-enhanced uptake of preformed fibrils of tau (pffs) by astroglial cells was through HSPG-mediated macropinocytosis, which was reduced by heparin treatment. pffs 
internalized by astroglial cells were delivered into lysosomes and degraded. In vivo studies showed that AAV-TFEB induction in astrocytes in a slowly progressing PS19 tauopathy mouse model reduced tau pathology and activation of glial cells, while this was not seen in the more aggressive rTg4510 model. In a pff-induced NFT transmission mouse model, pffs were injected into the ipsilateral side of the brain. Astroglial TFEB expression reduced tau pathology in the contralateral hippocampus at the early time point and in both ipsilateral and contralateral hippocampi at the later time point. These findings suggest astroglial TFEB plays an important role in the upregulation of extracellular tau degradation and the inhibition of tau transmission in tauopathies (Martini-Stoica et al., 2018).

\section{THERAPEUTIC PERSPECTIVES OF TAU DEGRADATION AND PROPAGATION BY AELN}

Understanding the molecular mechanisms that mediate the degradation and transmission of tau by AELN has a significant impact on the therapeutic interventions for different Tauopathies (Nixon, 2013; Li and Götz, 2017; Boland et al., 2018). The therapeutic strategy can be generally divided into two large categories: (1) Enhancing Tau degradation by AELN. (2) Preventing Tau Transmission through AELN.

\section{Enhancing Tau Degradation by AELN}

Dozens of drugs that can enhance tau degradation through AELN have been identified and tested in vitro and/or in vivo, as summarized in Table 5. Of notice, cilostazol, nilotinib, and curcumin are already in Phase II Clinical Trials.

Phase II clinical trial of lithium has just ended in January 2020, and the results are published on ClinicalTrials.gov (NCT02129348). Many patients with AD did not show any response or showed a minimal response to lithium for agitation, aggression, or psychosis. Some of them had intolerable side effects, including the increased risk of mortality (60-70\% higher than the placebo group), although the clinically used dose was 150-600 mg. A Brazilian group tested a micro-dose of lithium at $300 \mu \mathrm{g}$ in $\mathrm{AD}$ patients for 15 months and found no decreased performance, thus recommending the application of micro-dose lithium in $\mathrm{AD}$ clinical trials.

Leuco-methylthioninium bis (hydromethanesulphonate) (LMTM), which is a stable reduced derivative of methylene blue, has gone through four Phase III trials by TauRx. In the first three trials (NCT01689246 for mild to moderate AD patients, NCT01689233 for mild AD patients, and NCT01626378 for subjects with behavioral variant frontotemporal dementia (bvFTD)), patients who took LMTM at $100 \mathrm{mg}$ or $125 \mathrm{mg}$ twice a day as their only therapy performed significantly better on cognitive and functional tests than those who took $8 \mathrm{mg}$, although with small group size and questionable subset analysis (Gauthier et al., 2016; Wilcock et al., 2018). Further analysis from NCT01689246, NCT01689233, and NCT01626378 show that the lowest dose ( $8 \mathrm{mg} /$ day) of LMTM produced statistically significant concentration-dependent effects on the clinical decline and brain atrophy in both AD and bvFTD (Schelter et al., 2019; Shiells et al., 2020). In addition, TauRx recently finished a Phase III open-label extension trial (NCT02245568 in subjects with AD or bvFTD) for LMTM. TauRx is currently planning a new clinical trial, named LUCIDITY, where it uses FDG-PEG imaging and a composite cognitive/functional clinical psychometric scale to confirm the efficacy of LMTM as monotherapy at 8 and 16 mg/day. Another Phase II clinical trial (NCT02380573) for methylene blue in healthy aging, $\mathrm{MCI}$, and $\mathrm{AD}$ was being conducted by Texas Alzheimer's Research and Care Consortium. Unfortunately, this study has been suspended for now due to a funding issue.

Interestingly, VER-155008, a Hsc70 Chaperone inhibitor, did not inhibit PHF-tau degradation, rather increased reduction of PHF-tau, reversed axonal degeneration, and recovered memory function in 5xFAD mice, suggesting Hsc70 inhibition can lead to inhibition of UPS and CMA and shift to macroautophagy pathway. However, macroautophagy activation was not examined in that study (Yang and Tohda, 2018).

Another important aspect for enhancing tau removal is to promote tau degradation inside neurons or microglia using tau antibodies through active or passive immunotherapies [reviewed in Congdon and Sigurdsson (2018)]. It has been shown that anti-tau antibodies can enter neurons and co-localize with endosomes and lysosomes. Our group has recently reported that utilizing the Virus-like Particle (VLP) platform to generate vaccines targeting pT181 tau can induce a robust and longlasting anti-pT181 antibody response in the sera and the brains of both non-transgenic and rTG4510 mouse model of FTD (Maphis et al., 2019). pT181-Qß vaccination reduced both soluble and insoluble pTau in the hippocampus and cortex, prevented hippocampal and corpus callosum atrophy, and rescued cognitive dysfunction in 4-month -old rTg4510 mice. This study proposes a VLP vaccine-based approach in the prevention and treatment of tauopathies. Although the exact mechanisms remain to be elucidated, it has been proposed that anti-tau antibodies can bind to extracellular tau and enter neurons through bulk endocytosis or receptor-mediated endocytosis and degrade tau through AELN (Congdon and Sigurdsson, 2018).

Microglia have also been shown to efficiently phagocytose and degrade AT8-positive human tau that is passively released from unfixed frozen brain sections of P301L transgenic mice and human AD brain sections and remove NFTs from P301S tauopathy mouse brain sections (Luo et al., 2015). Microglial phagocytosis of tau was enhanced by anti-tau monoclonal antibody MC1 - specific for conformational epitopes on PHF tau in an Fc $\gamma$-receptor-dependent manner. Another study also confirmed an anti-pS396-tau antibody C10.2-mediated clearance of tau in primary mouse microglial cultures needs Fc $\gamma$-receptor binding for antibody uptake and requires effector function and functional lysosome for internalized antibody-bound tau degradation (Andersson et al., 2019). Although another study argued that antibody effector function and microglia involvement is not required for antibody-mediated targeting of tau in vivo since only full-effector antibody promoted tau uptake into microglia and subsequently induced proinflammatory cytokine 
TABLE 5 | Approaches to enhance tau degradation by AELN.

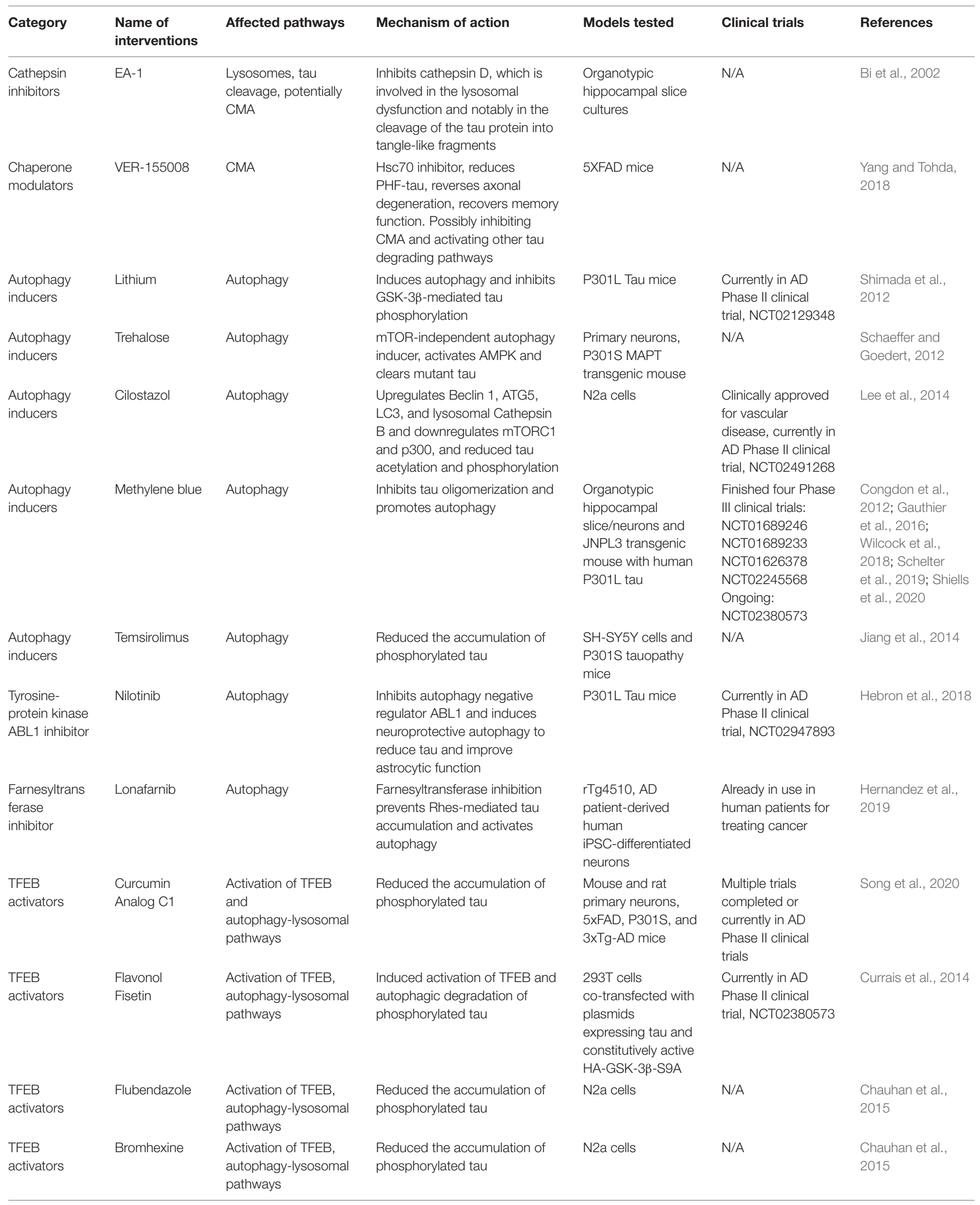


TABLE 5 | Continued

\begin{tabular}{|c|c|c|c|c|c|c|}
\hline Category & $\begin{array}{l}\text { Name of } \\
\text { interventions }\end{array}$ & Affected pathways & Mechanism of action & Models tested & Clinical trials & References \\
\hline $\begin{array}{l}\text { TFEB } \\
\text { activators }\end{array}$ & Optogenetics & $\begin{array}{l}\text { Activation of TFEB, } \\
\text { autophagy-lysosomal } \\
\text { pathways }\end{array}$ & $\begin{array}{l}\text { Reduced the accumulation of } \\
\text { phosphorylated tau }\end{array}$ & $\begin{array}{l}\text { N2a cells, AD } \\
\text { patient-derived } \\
\text { human } \\
\text { iPSC-differentiated } \\
\text { neurons }\end{array}$ & $\mathrm{N} / \mathrm{A}$ & Binder et al., 2020 \\
\hline $\begin{array}{l}\text { Tau } \\
\text { antibodies }\end{array}$ & $\begin{array}{l}\text { Various } \\
\text { antibodies }\end{array}$ & $\begin{array}{l}\text { Lysosomal } \\
\text { degradative } \\
\text { pathways }\end{array}$ & $\begin{array}{l}\text { Reduce oligomerization of tau } \\
\text { and enhance tau degradation } \\
\text { through AELN inside neurons or } \\
\text { microglia }\end{array}$ & $\begin{array}{l}\text { Various transgenic } \\
\text { mice }\end{array}$ & $\begin{array}{l}\text { Currently in Phase I } \\
\text { or II AD clinical trials }\end{array}$ & $\begin{array}{l}\text { Congdon and } \\
\text { Sigurdsson, } 2018\end{array}$ \\
\hline
\end{tabular}

TABLE 6 | Approaches to prevent tau transmission through AELN.

\begin{tabular}{|c|c|c|c|c|c|}
\hline Category & $\begin{array}{l}\text { Name of } \\
\text { interventions }\end{array}$ & Affected pathways & Mechanism of action & Models tested & References \\
\hline $\begin{array}{l}\text { Tau acetylation } \\
\text { inhibitor }\end{array}$ & $\begin{array}{l}\text { p300 Inhibitor } \\
37892\end{array}$ & $\begin{array}{l}\text { Degradative Autophagy, } \\
\text { tau secretion (unknown } \\
\text { pathway) }\end{array}$ & $\begin{array}{l}\text { Inhibits p300/CBP, reduces tau acetylation, } \\
\text { increases autophagy flux, and dampens tau } \\
\text { secretion }\end{array}$ & $\begin{array}{l}\text { Tau fibrils and AAV-Cre or AAV-GFP } \\
\text { into 4-5-month-old PS19 mice } \\
\text { carrying } \mathrm{p} 300^{\mathrm{F} / \mathrm{F}} \text { and } \mathrm{CBP} / \mathrm{F}\end{array}$ & Chen et al., 2020 \\
\hline Tau antibodies & $\begin{array}{l}\text { Various } \\
\text { antibodies }\end{array}$ & $\begin{array}{l}\text { Tau uptake and } \\
\text { transmission }\end{array}$ & $\begin{array}{l}\text { Prevent tau uptake and neuron-to-neuron } \\
\text { transmission }\end{array}$ & $\begin{array}{l}\text { Microfluidic chamber and primary } \\
\text { mouse neurons }\end{array}$ & $\begin{array}{l}\text { Nobuhara et al., } \\
2017\end{array}$ \\
\hline $\begin{array}{l}\text { nSMase2 } \\
\text { inhibitor }\end{array}$ & Cambinol & $\begin{array}{l}\text { Extracellular } \\
\text { Vesicle/Exosome }\end{array}$ & $\begin{array}{l}\text { Suppresses extracellular vesicle (EV) } \\
\text { production while reducing tau seed } \\
\text { propagation }\end{array}$ & $\mathrm{N} 2 \mathrm{a}$ cells & $\begin{array}{l}\text { Bilousova et al., } \\
2018\end{array}$ \\
\hline HSPG inhibition & Heparin & $\begin{array}{l}\text { HSPG-mediated tau } \\
\text { secretion, uptake, and } \\
\text { transmission }\end{array}$ & $\begin{array}{l}\text { Reduced tau secretion from neurons and tau } \\
\text { uptake by neurons and astrocytes }\end{array}$ & Primary mouse astrocytes & $\begin{array}{l}\text { Martini-Stoica } \\
\text { et al., } 2018\end{array}$ \\
\hline
\end{tabular}

secretion, which can be detrimental to neurons, thus proposing effectorless tau antibodies might be safer for a therapeutic purpose (Lee et al., 2016).

\section{Preventing Tau Transmission Through AELN}

Since neuron-to-neuron, neuron-to-glia tau transmission is an emerging field; there have not been many attempts yet to prevent tau transmission.

It has been shown that various antibodies targeting different regions of tau can exhibit a differential inhibitory effect on tau uptake and transmission between neurons. Antibodies targeting $\mathrm{N}$-terminal (Tau13) and Mid-domain of tau (6C5 and HT7) can prevent internalization of tau, while antibody targeting C-terminal tau cannot. Phosphorylation-dependent (40E8 and p396) and C-terminal half (4E4) antibodies can also reduce tau internalization, although to a lesser extent. $6 \mathrm{C} 5$, the mid-domain targeting antibody, was able to block tau uptake and neuron-toneuron propagation of tau in a microfluidic device and primary mouse neurons. It will be interesting to see if these antibodies have the same effects in vivo (Nobuhara et al., 2017).

Based on the current literature on AELN-mediated tau propagation, potential therapeutic interventions for preventing tau transmission can be summarized as follows (Table 6):

(1) p300 inhibitor 37892, a small molecule inhibitor identified through screening, has been shown to inhibit p300/CBP, prevent tau acetylation, promote tau degradation through autophagy, and reduce tau secretion through an unidentified pathway.

(2) Cambinol, a nSMase2 inhibitor, was shown to suppress extracellular vesicle formation and tau secretion.

(3) Boosting astroglial TFEB activation since astroglial TFEB was involved in attenuating extracellular tau propagation and upregulating extracellular tau degradation.

(4) Inhibiting microglial BIN1 isoform 9 since it favors tau spreading.

(5) Inhibiting HSPG through Heparin, considering the involvement of HSPG in tau secretion, uptake, and transmission in neurons and astrocytes.

\section{DISCUSSION}

The degradation and propagation of tau through AELN is a complex process, and new studies are emerging to rapidly change the current state of knowledge.

Different isoforms and various post-translationally modified tau favor certain degradative pathways inside neurons, and different strains of tau also exhibit the distinct capacity of propagation in tauopathies. Considering the heterogenicity within tauopathies or even within the single patient, precision medicine needs to be taken into consideration when determining the therapeutic strategy for tauopathy patients.

On the other hand, autophagy plays a dual role in tau pathology: First, degrading pathological tau inside neurons and microglia; Second, mediating the unconventional secretion of 
phosphorylated tau, although autophagy-based unconventional secretion of tau needs further investigation both in vitro and in vivo.

In addition, neuronal and microglial BIN1 isoforms seem to have opposite functions. Neuronal BIN1 inhibits RAB5 activation and endosome formation to reduce tau propagation inside neurons, while microglial BIN1 isoform 9 is shown to promote tau propagation by incorporating tau into exosomes.

There are also multiple molecular pathways mediating tau release from neurons and tau internalization into neurons and glial cells. Therefore, investigating the molecular pathways mediating the degradation and transmission of the most neurotoxic form of tau by AELN will be beneficial to finding the novel therapeutic targets in AD and related tauopathies.

In this review, we attempted to list the potential targets and those that are already being examined in clinical trials, including tau-targeting antibodies. The literature on different modes of antibody-mediated clearance is a vast topic, and the goal of this review is not to go into the depth of any one type of mechanism in detail. Rather, we attempted to provide a generic overview

\section{REFERENCES}

Andersson, C. R., Falsig, J., Stavenhagen, J. B., Christensen, S., Kartberg, F., Rosenqvist, N., et al. (2019). Antibody-mediated clearance of tau in primary mouse microglial cultures requires $\mathrm{Fc} \gamma$-receptor binding and functional lysosomes. Sci. Rep. 9, 1-12. doi: 10.1038/s41598-019-41105-4

Ando, K., Brion, J. P., Stygelbout, V., Suain, V., Authelet, M., Dedecker, R., et al. (2013). Clathrin adaptor CALM/PICALM is associated with neurofibrillary tangles and is cleaved in Alzheimer's brains. Acta Neuropathol. 125, 861-878. doi: 10.1007/s00401-013-1111-z

Ando, K., Tomimura, K., Sazdovitch, V., Suain, V., Yilmaz, Z., Authelet, M., et al. (2016). Level of PICALM, a key component of clathrin-mediated endocytosis, is correlated with levels of phosphotau and autophagy-related proteins and is associated with tau inclusions in AD, PSP and Pick disease. Neurobiol. Dis. 94 32-43. doi: 10.1016/j.nbd.2016.05.017

Asai, H., Ikezu, S., Tsunoda, S., Medalla, M., Luebke, J., Haydar, T., et al. (2015). Depletion of microglia and inhibition of exosome synthesis halt tau propagation. Nat. Neurosci. 18, 1584-1593. doi: 10.1038/nn. 4132

Avila, J., Gómez De Barreda, E., Engel, T., Lucas, J. J., and Hernández, F. (2010). Tau phosphorylation in hippocampus results in toxic gain-of-function. Biochem. Soc. Trans. 38, 977-980.

Bi, X., Haque, T. S., Zhou, J., Skillman, A. G., Lin, B., Lee, C. E., et al. (2002). Novel cathepsin D inhibitors block the formation of hyperphosphorylated tau fragments in hippocampus. J. Neurochem. 74, 1469-1477. doi: 10.1046/j.14714159.2000.0741469.x

Bilousova, T., Elias, C., Miyoshi, E., Alam, M. P., Zhu, C., Campagna, J., et al. (2018). Suppression of tau propagation using an inhibitor that targets the DK-switch of nSMase2. Biochem. Biophys. Res. Commun. 499, 751-757. doi: 10.1016/j.bbrc.2018.03.209

Binder, J. L., Chander, P., Deretic, V., Weick, J. P., and Bhaskar, K. (2020). Optical induction of autophagy via Transcription factor EB (TFEB) reduces pathological tau in neurons. PLoS One 15:e0230026. doi: 10.1371/journal.pone. 0230026

Boland, B., Yu, W. H., Corti, O., Mollereau, B., Henriques, A., Bezard, E., et al. (2018). Promoting the clearance of neurotoxic proteins in neurodegenerative disorders of ageing. Nat. Rev. Drug Discov. 17, 660-688. doi: 10.1038/nrd. 2018.109

Braak, H., and Braak, E. (1995). Staging of alzheimer's disease-related neurofibrillary changes. Neurobiol. Aging 16, 271-278. doi: 10.1016/01974580(95)00021-6 of the current status of all the studies relevant to this topic and encourage readers to refer to other relevant reviews on specific topics.

\section{AUTHOR CONTRIBUTIONS}

SJ and KB wrote the manuscript. Both authors contributed to the article and approved the submitted version.

\section{FUNDING}

This study was supported by NIH/NINDS R01NS083704 and RF1NS083704 (KB) and P20GM121176.

\section{ACKNOWLEDGMENTS}

Figures are created with BioRender.com

Caballero, B., Wang, Y., Diaz, A., Tasset, I., Juste, Y. R., Stiller, B., et al. (2018). Interplay of pathogenic forms of human tau with different autophagic pathways. Aging Cell 17:e12692. doi: 10.1111/acel.12692

Calafate, S., Flavin, W., Verstreken, P., and Moechars, D. (2016). Loss of bin1 promotes the propagation of tau pathology. Cell Rep. 17, 931-940. doi: 10.1016/ j.celrep.2016.09.063

Chang, S. H., Jung, I. S., Han, G. Y., Kim, N. H., Kim, H. J., and Kim, C. W. (2013). Proteomic profiling of brain cortex tissues in a Tau transgenic mouse model of Alzheimer's disease. Biochem. Biophys. Res. Commun. 430, 670-675. doi: 10.1016/j.bbrc.2012.11.093

Chauhan, S., Ahmed, Z., Bradfute, S. B., Arko-Mensah, J., Mandell, M. A., Won Choi, S., et al. (2015). Pharmaceutical screen identifies novel target processes for activation of autophagy with a broad translational potential. Nat. Commun. 6:8620. doi: $10.1038 /$ ncomms 9620

Chauhan, S., Goodwin, J. G., Chauhan, S., Manyam, G., Wang, J., Kamat, A. M., et al. (2013). ZKSCAN3 is a master transcriptional repressor of autophagy. Mol. Cell 50, 16-28. doi: 10.1016/j.molcel.2013.01.024

Chen, X., Li, Y., Wang, C., Tang, Y., Mok, S.-A., Tsai, R. M., et al. (2020). Promoting tau secretion and propagation by hyperactive p300/CBP via autophagylysosomal pathway in tauopathy. Mol. Neurodegen. 15:2. doi: 10.1186/s13024019-0354-0

Clavaguera, F., Bolmont, T., Crowther, R. A., Abramowski, D., Frank, S., Probst, A., et al. (2009). Transmission and spreading of tauopathy in transgenic mouse brain. Nat. Cell Biol. 11, 909-913. doi: 10.1038/ncb1901

Cocucci, E., and Meldolesi, J. (2015). Ectosomes and exosomes: shedding the confusion between extracellular vesicles. Trends Cell Biol. 25, 364-372. doi: 10.1016/j.tcb.2015.01.004

Colacurcio, D. J., and Nixon, R. A. (2016). Disorders of lysosomal acidificationThe emerging role of $\mathrm{v}$-ATPase in aging and neurodegenerative disease. Ageing Res. Rev. 32, 75-88. doi: 10.1016/j.arr.2016.05.004

Congdon, E. E., and Sigurdsson, E. M. (2018). Tau-targeting therapies for Alzheimer disease. Nat. Rev. Neurol. 14, 399-415. doi: 10.1038/s41582-0180013-z

Congdon, E. E., Wu, J. W., Myeku, N., Figueroa, Y. H., Herman, M., Marinec, P. S., et al. (2012). Methylthioninium chloride (methylene blue) induces autophagy and attenuates tauopathy in vitro and in vivo. Autophagy 8, 609-622. doi: 10.4161/auto. 19048

Coppola, G., Chinnathambi, S., Lee, J. J., Dombroski, B. A., Baker, M. C., SotoOrtolaza, A. I., et al. (2012). Evidence for a role of the rare p.A152T variant in MAPT in increasing the risk for FTD-spectrum and Alzheimer's diseases. Hum. Mol. Genet. 21, 3500-3512. doi: 10.1093/hmg/dds161 
Crotti, A., Sait, H. R., McAvoy, K. M., Estrada, K., Ergun, A., Szak, S., et al. (2019). BIN1 favors the spreading of Tau via extracellular vesicles. Sci. Rep. 9, 1-20. doi: 10.1038/s41598-019-45676-0

Cuervo, A. M., and Wong, E. (2014). Chaperone-mediated autophagy: roles in disease and aging. Cell Res. 24, 92-104. doi: 10.1038/cr.2013.153

Currais, A., Prior, M., Dargusch, R., Armando, A., Ehren, J., Schubert, D., et al. (2014). Modulation of p25 and inflammatory pathways by fisetin maintains cognitive function in Alzheimer's disease transgenic mice. Aging Cell 13, 379390. doi: 10.1111/acel.12185

Dall'Armi, C., Hurtado-Lorenzo, A., Tian, H., Morel, E., Nezu, A., Chan, R. B., et al. (2010). The phospholipase D1 pathway modulates macroautophagy. Nat. Commun. 1, 1-11. doi: 10.1038/ncomms1144

De Calignon, A., Polydoro, M., Suárez-Calvet, M., William, C., Adamowicz, D. H., Kopeikina, K. J., et al. (2012). Propagation of tau pathology in a model of early Alzheimer's disease. Neuron 73, 685-697. doi: 10.1016/j.neuron.2011.11.033

Dice, J. F. (1982). Altered degradation of proteins microinjected into senescent human fibroblasts. J. Biol. Chem. 257, 14624-14627.

Dickson, D. W., Baker, M., and Rademakers, R. (2010). Common variant in GRN is a genetic risk factor for hippocampal sclerosis in the elderly. Neurodegen. Dis. 7, 170-174. doi: 10.1159/000289231

Dikic, I. (2017). Proteasomal and autophagic degradation systems. Annu. Rev. Biochem. 86, 193-224. doi: 10.1146/annurev-biochem-061516-044908

Dixit, R., Ross, J. L., Goldman, Y. E., and Holzbaur, E. L. F. (2008). Differential regulation of dynein and kinesin motor proteins by tau. Science 319, 1086-1089. doi: 10.1126/science. 1152993

Dujardin, S., Bégard, S., Caillierez, R., Lachaud, C., Delattre, L., Carrier, S., et al. (2014). Ectosomes: a new mechanism for non-exosomal secretion of tau protein. PLoS One 9:e100760. doi: 10.1371/journal.pone.0100760

Dupont, N., Jiang, S., Pilli, M., Ornatowski, W., Bhattacharya, D., and Deretic, V. (2011). Autophagy-based unconventional secretory pathway for extracellular delivery of IL-1 beta. EMBO J. 30, 4701-4711. doi: 10.1038/emboj.2011.398

Falcon, B., Noad, J., McMahon, H., Randow, F., and Goedert, M. (2018a). Galectin8-mediated selective autophagy protects against seeded tau aggregation. J. Biol. Chem. 293, 2438-2451. doi: 10.1074/jbc.M117.809293

Falcon, B., Zhang, W., Murzin, A. G., Murshudov, G., Garringer, H. J., Vidal, R., et al. (2018b). Structures of filaments from Pick's disease reveal a novel tau protein fold. Nature 561, 137-140. doi: 10.1038/s41586-018-0454-y

Falcon, B., Zivanov, J., Zhang, W., Murzin, A. G., Garringer, H. J., Vidal, R., et al. (2019). Novel tau filament fold in chronic traumatic encephalopathy encloses hydrophobic molecules. Nature 568, 420-423. doi: 10.1038/s41586-019-1026-5

Farfel-Becker, T., Roney, J. C., Cheng, X.-T., Li, S., Cuddy, S. R., and Sheng, Z.-H. (2019). Neuronal soma-derived degradative lysosomes are continuously delivered to distal axons to maintain local degradation capacity. Cell Rep. 28, 51.e4-64.e4. doi: 10.1016/j.celrep.2019.06.013

Farg, M. A., Sundaramoorthy, V., Sultana, J. M., Yang, S., Atkinson, R. A. K., Levina, V., et al. (2014). C9ORF72, implicated in amytrophic lateral sclerosis and frontotemporal dementia, regulates endosomal trafficking. Hum. Mol. Genet. 23, 3579-3595. doi: 10.1093/hmg/ddu068

Ferrer, I., Aguiló García, M., Carmona, M., Andrés-Benito, P., Torrejón-Escribano, B., Garcia-Esparcia, P., et al. (2019). Involvement of oligodendrocytes in tau seeding and spreading in tauopathies. Front. Aging Neurosci. 11:112. doi: 10. 3389/fnagi.2019.00112

Fitzpatrick, A. W. P., Falcon, B., He, S., Murzin, A. G., Murshudov, G., Garringer, H. J., et al. (2017). Cryo-EM structures of tau filaments from Alzheimer's disease. Nature 547, 185-190. doi: 10.1038/nature23002

Frost, B., Jacks, R. L., and Diamond, M. I. (2009). Propagation of Tau misfolding from the outside to the inside of a cell. J. Biol. Chem. 284, 12845-12852. doi: 10.1074/jbc.M808759200

Fuster-Matanzo, A., Hernández, F., and Ávila, J. (2018). Tau spreading mechanisms; Implications for dysfunctional tauopathies. Int. J. Mol. Sci. 19:645. doi: 10.3390/ijms19030645

Gauthier, S., Feldman, H. H., Schneider, L. S., Wilcock, G. K., Frisoni, G. B., Hardlund, J. H., et al. (2016). Efficacy and safety of tau-aggregation inhibitor therapy in patients with mild or moderate Alzheimer's disease: a randomised, controlled, double-blind, parallel-arm, phase 3 trial. Lancet 388, 2873-2884. doi: 10.1016/S0140-6736(16)31275-2

Gee, H. Y., Noh, S. H., Tang, B. L., Kim, K. H., and Lee, M. G. (2011). Rescue of delta F508-CFTR trafficking via a GRASP-dependent unconventional secretion pathway. Cell 146, 746-760. doi: 10.1016/j.cell.2011.07.021
Gibbons, G. S., Lee, V. M. Y., and Trojanowski, J. Q. (2019). Mechanisms of cellto-cell transmission of pathological tau: a review. JAMA Neurol. 76, 101-108. doi: 10.1001/jamaneurol.2018.2505

Götz, J., Halliday, G., and Nisbet, R. M. (2019). Molecular Pathogenesis of the Tauopathies. Annu. Rev. Pathol. Mech. Dis. 14, 239-261. doi: 10.1146/annurevpathmechdis-012418-012936

Guillozet-Bongaarts, A. L., Glajch, K. E., Libson, E. G., Cahill, M. E., Bigio, E., Berry, R. W., et al. (2007). Phosphorylation and cleavage of tau in non-AD tauopathies. Acta Neuropathol. 113, 513-520. doi: 10.1007/s00401-007-0209-6

Guo, J. L., and Lee, V. M. Y. (2011). Seeding of normal tau by pathological tau conformers drives pathogenesis of Alzheimer-like tangles. J. Biol. Chem. 286, 15317-15331. doi: 10.1074/jbc.M110.209296

Guo, J. L., Narasimhan, S., Changolkar, L., He, Z., Stieber, A., Zhang, B., et al. (2016). Unique pathological tau conformers from alzheimer's brains transmit tau pathology in nontransgenic mice. J. Exp. Med. 213, 2635-2654. doi: 10.1084/ jem. 20160833

Hamano, T., Gendron, T. F., Causevic, E., Yen, S. H., Lin, W. L., Isidoro, C., et al. (2008). Autophagic-lysosomal perturbation enhances tau aggregation in transfectants with induced wild-type tau expression. Eur. J. Neurosci. 27, 1119-1130. doi: 10.1111/j.1460-9568.2008.06084.x

Hebron, M. L., Javidnia, M., and Moussa, C. E. H. (2018). Tau clearance improves astrocytic function and brain glutamate-glutamine cycle. J. Neurol. Sci. 391, 90-99. doi: 10.1016/j.jns.2018.06.005

Hernandez, I., Luna, G., Rauch, J. N., Reis, S. A., Giroux, M., Karch, C. M., et al. (2019). A farnesyltransferase inhibitor activates lysosomes and reduces tau pathology in mice with tauopathy. Sci. Transl. Med. 11:eaat3005. doi: 10.1126/ scitranslmed.aat3005

Himmler, A., Drechsel, D., Kirschner, M. W., and Martin, D. W. (1989). Tau consists of a set of proteins with repeated C-terminal microtubule-binding domains and variable N-terminal domains. Mol. Cell. Biol. 9, 1381-1388. doi: 10.1128/mcb.9.4.1381

Holler, C. J., Davis, P. R., Beckett, T. L., Platt, T. L., Webb, R. L., Head, E., et al. (2014). Bridging integrator 1 (BIN1) protein expression increases in the alzheimer's disease brain and correlates with neurofibrillary tangle pathology. J. Alzheimers Dis. 42, 1221-1227. doi: 10.3233/JAD-132450

Holmes, B. B., DeVos, S. L., Kfoury, N., Li, M., Jacks, R., Yanamandra, K., et al. (2013). Heparan sulfate proteoglycans mediate internalization and propagation of specific proteopathic seeds. Proc. Natl. Acad. Sci. U.S.A. 110, E3138-E3147. doi: $10.1073 /$ pnas. 1301440110

Hutton, M., Lendon, C. L., Rizzu, P., Baker, M., Froelich, S., Houlden, H. H., et al. (1998). Association of missense and 5'-splice-site mutations in tau with the inherited dementia FTDP-17. Nature 393, 702-704. doi: 10.1038/31508

Iba, M., Guo, J. L., McBride, J. D., Zhang, B., Trojanowski, J. Q., and Lee, V. M. Y. (2013). Synthetic tau fibrils mediate transmission of neurofibrillary tangles in a transgenic mouse model of alzheimer's-like tauopathy. J. Neurosci. 33, 1024-1037. doi: 10.1523/JNEUROSCI.2642-12.2013

Ikeda, K., Akiyama, H., Arai, T., Kondo, H., Haga, C., Iritani, S., et al. (1998). Alz50/Gallyas-positive lysosome-like intraneuronal granules in Alzheimer's disease and control brains. Neurosci. Lett. 258, 113-116. doi: 10.1016/S0304-3940(98) 00867-2

Ikeda, K., Akiyama, H., Arai, T., Kondo, H., Haga, C., Tsuchiya, K., et al. (2000). Neurons containing Alz-50-immunoreactive granules around the cerebral infarction: evidence for the lysosomal degradation of altered tau in human brain? Neurosci. Lett. 284, 187-189. doi: 10.1016/S0304-3940(00)01009-0

Iqbal, K., Liu, F., and Gong, C.-X. (2016). Tau and Neurodegenerative Disease: The Story So Far. Berlin: Nature Publishing Group.

Jiang, T., Yu, J. T., Zhu, X. C., Zhang, Q. Q., Cao, L., Wang, H. F., et al. (2014). Temsirolimus attenuates tauopathy in vitro and in vivo by targeting tau hyperphosphorylation and autophagic clearance. Neuropharmacology 85, 121-130. doi: 10.1016/j.neuropharm.2014.05.032

Johansen, T., and Lamark, T. (2020). Selective autophagy: ATG8 family proteins, LIR motifs and cargo receptors. J. Mol. Biol. 432, 80-103. doi: 10.1016/j.jmb. 2019.07.016

Kang, S., Son, S. M., Baik, S. H., Yang, J., and Mook-Jung, I. (2019). Autophagymediated secretory pathway is responsible for both normal and pathological tau in neurons. J. Alzheimers Dis. JAD 70, 667-680. doi: 10.3233/JAD-190180

Katsinelos, T., Zeitler, M., Dimou, E., Karakatsani, A., Müller, H. M., Nachman, E., et al. (2018). Unconventional secretion mediates the trans-cellular spreading of tau. Cell Rep. 23, 2039-2055. doi: 10.1016/j.celrep.2018.04.056 
Katsumata, K., Nishiyama, J., Inoue, T., Mizushima, N., Takeda, J., and Yuzaki, M. (2010). Dynein-and activity-dependent retrograde transport of autophagosomes in neuronal axons. Autophagy 6, 378-385. doi: 10.4161/auto. 6.3.11262

Kaushik, S., and Cuervo, A. M. (2018). The coming of age of chaperone-mediated autophagy. Nat. Rev. Mol. Cell Biol. 19, 365-381. doi: 10.1038/s41580-0180001-6

Kraus, A., Saijo, E., Metrick, M. A., Newell, K., Sigurdson, C. J., Zanusso, G., et al. (2019). Seeding selectivity and ultrasensitive detection of tau aggregate conformers of Alzheimer disease. Acta Neuropathol. 137, 585-598. doi: 10.1007/ s00401-018-1947-3

Krüger, U., Wang, Y., Kumar, S., and Mandelkow, E.-M. (2012). Autophagic degradation of tau in primary neurons and its enhancement by trehalose. Neurobiol. Aging 33, 2291-2305. doi: 10.1016/J.NEUROBIOLAGING.2011. 11.009

Lee, H. R., Shin, H. K., Park, S. Y., Kim, H. Y., Lee, W. S., Rhim, B. Y., et al. (2014). Attenuation of $\beta$-amyloid-induced tauopathy via activation of CK2 $\alpha /$ SIRT1: targeting for cilostazol. J. Neurosci. Res. 92, 206-217. doi: 10.1002/jnr. 23310

Lee, M. J., Lee, J. H., and Rubinsztein, D. C. (2013). Tau degradation: the ubiquitinproteasome system versus the autophagy-lysosome system. Prog. Neurobiol. 105, 49-59. doi: 10.1016/j.pneurobio.2013.03.001

Lee, S. H., Le Pichon, C. E., Adolfsson, O., Gafner, V., Pihlgren, M., Lin, H., et al. (2016). Antibody-mediated targeting of tau in vivo does not require effector function and microglial engagement. Cell Rep. 16, 1690-1700. doi: 10.1016/j. celrep.2016.06.099

Lee, V. M.-Y., Goedert, M., and Trojanowski, J. Q. (2001). Neurodegenerative tauopathies. Annu. Rev. Neurosci. 24, 1121-1159. doi: 10.1146/annurev.neuro. 24.1.1121

Li, C., and Götz, J. (2017). Tau-based therapies in neurodegeneration: opportunities and challenges. Nat. Rev. Drug Discov. 16, 863-883. doi: 10.1038/nrd.2017.155

Liu, L., Drouet, V., Wu, J. W., Witter, M. P., Small, S. A., Clelland, C., et al. (2012). Trans-synaptic spread of tau pathology in vivo. PLoS One 7:e0031302. doi: 10.1371/journal.pone.0031302

Luo, W., Liu, W., Hu, X., Hanna, M., Caravaca, A., and Paul, S. M. (2015). Microglial internalization and degradation of pathological tau is enhanced by an anti-tau monoclonal antibody. Sci. Rep. 5:11161. doi: 10.1038/srep11161

Maphis, N., Xu, G., Kokiko-Cochran, O. N., Jiang, S., Cardona, A., Ransohoff, R. M., et al. (2015). Reactive microglia drive tau pathology and contribute to the spreading of pathological tau in the brain. Brain J. Neurol. 138(Pt 6), 1738-1755. doi: 10.1093/brain/awv081

Maphis, N. M., Peabody, J., Crossey, E., Jiang, S., Jamaleddin Ahmad, F. A., Alvarez, M., et al. (2019). Qß Virus-like particle-based vaccine induces robust immunity and protects against tauopathy. NPJ Vaccines 4:26. doi: 10.1038/s41541-0190118-4

Martini-Stoica, H., Cole, A. L., Swartzlander, D. B., Chen, F., Wan, Y. W., Bajaj, L., et al. (2018). TFEB enhances astroglial uptake of extracellular tau species and reduces tau spreading. J. Exp. Med. 215, 2355-2377. doi: 10.1084/jem.2017 2158

Menzies, F. M., Fleming, A., Caricasole, A., Bento, C. F., Andrews, S. P., Ashkenazi, A., et al. (2017). Autophagy and neurodegeneration: pathogenic mechanisms and therapeutic opportunities. Neuron 93, 1015-1034. doi: 10.1016/j.neuron. 2017.01.022

Merezhko, M., Brunello, C. A., Yan, X., Vihinen, H., Jokitalo, E., Uronen, R. L., et al. (2018). Secretion of tau via an unconventional non-vesicular mechanism. Cell Rep. 25, 2027.e4-2035.e4. doi: 10.1016/j.celrep.2018.10.078

Min, S. W., Cho, S. H., Zhou, Y., Schroeder, S., Haroutunian, V., Seeley, W. W., et al. (2010). Acetylation of tau inhibits its degradation and contributes to tauopathy. Neuron 67, 953-966. doi: 10.1016/j.neuron.2010.08.044

Moreau, K., Fleming, A., Imarisio, S., Lopez Ramirez, A., Mercer, J. L., JimenezSanchez, M., et al. (2014). PICALM modulates autophagy activity and tau accumulation. Nat. Commun. 5:4998. doi: 10.1038/ncomms5998

Narasimhan, S., Guo, J. L., Changolkar, L., Stieber, A., McBride, J. D., Silva, L. V., et al. (2017). Pathological tau strains from human brains recapitulate the diversity of tauopathies in nontransgenic mouse brain. J. Neurosci. 37, 11406-11423. doi: 10.1523/JNEUROSCI.1230-17.2017

Nixon, R. A. (2013). The role of autophagy in neurodegenerative disease. Nat. Med. 19, 983-997. doi: 10.1038/nm.3232
Nixon, R. A., Wegiel, J., Kumar, A., Yu, W. H., Peterhoff, C., Cataldo, A., et al. (2005). Extensive involvement of autophagy in alzheimer disease: an immunoelectron microscopy study. J. Neuropathol. Exp. Neurol. 64, 113-122. doi: 10. 1093/JNEN/64.2.113

Nobuhara, C. K., DeVos, S. L., Commins, C., Wegmann, S., Moore, B. D., Roe, A. D., et al. (2017). Tau antibody targeting pathological species blocks neuronal uptake and interneuron propagation of tau in vitro. Am. J. Pathol. 187, 13991412. doi: 10.1016/j.ajpath.2017.01.022

Paushter, D. H., Du, H., Feng, T., and Hu, F. (2018). The lysosomal function of progranulin, a guardian against neurodegeneration. Acta Neuropathol. 136:1. doi: 10.1007/s00401-018-1861-8

Perea, J. R., López, E., Díez-Ballesteros, J. C., Ávila, J., Hernández, F., and Bolós, M. (2019). Extracellular monomeric tau is internalized by astrocytes. Front. Neurosci. 13:442. doi: 10.3389/fnins.2019.00442

Perez, S. E., He, B., Nadeem, M., Wuu, J., Ginsberg, S. D., Ikonomovic, M. D., et al. (2015). Hippocampal endosomal, lysosomal, and autophagic dysregulation in mild cognitive impairment: correlation with $\mathrm{A} \beta$ and tau pathology. J. Neuropathol. Exp. Neurol. 74, 345-358. doi: 10.1097/NEN. 0000000000000179

Perry, D. C., Lehmann, M., Yokoyama, J. S., Karydas, A., Lee, J. J. Y., Coppola, G., et al. (2013). Progranulin mutations as risk factors for alzheimer disease. JAMA Neurol. 70, 774-778. doi: 10.1001/2013.jamaneurol.393

Piras, A., Collin, L., Grüninger, F., Graff, C., and Rönnbäck, A. (2016). Autophagic and lysosomal defects in human tauopathies: analysis of post-mortem brain from patients with familial Alzheimer disease, corticobasal degeneration and progressive supranuclear palsy. Acta Neuropathol. Commun. 4:22. doi: 10.1186/ s40478-016-0292-9

Polito, V. A., Li, H., Martini-Stoica, H., Wang, B., Yang, L., Xu, Y., et al. (2014). Selective clearance of aberrant tau proteins and rescue of neurotoxicity by transcription factor EB. EMBO Mol. Med. 6, 1142-1160. doi: 10.15252/emmm. 201303671

Pooler, A. M., Phillips, E. C., Lau, D. H. W., Noble, W., and Hanger, D. P. (2013). Physiological release of endogenous tau is stimulated by neuronal activity. EMBO Rep. 14, 389-394. doi: 10.1038/embor.2013.15

Puangmalai, N., Bhatt, N., Montalbano, M., Sengupta, U., Gaikwad, S., Ventura, F., et al. (2020). Internalization mechanisms of brain-derived tau oligomers from patients with Alzheimer's disease, progressive supranuclear palsy and dementia with Lewy bodies. Cell Death Dis. 11:314. doi: 10.1038/s41419-0202503-3

Rapoport, M., Dawson, H. N., Binder, L. I., Vitek, M. P., and Ferreira, A. (2002). Tau is essential to $\beta$-amyloid-induced neurotoxicity. Proc. Natl. Acad. Sci. U.S.A. 99, 6364-6369. doi: 10.1073/pnas.092136199

Rodríguez-Navarro, J. A., Rodríguez, L., Casarejos, M. J., Solano, R. M., Gómez, A., Perucho, J., et al. (2010). Trehalose ameliorates dopaminergic and tau pathology in parkin deleted/tau overexpressing mice through autophagy activation. Neurobiol. Dis. 39, 423-438. doi: 10.1016/j.nbd.2010. 05.014

Sahu, R., Kaushik, S., Clement, C. C., Cannizzo, E. S., Scharf, B., Follenzi, A., et al. (2011). Microautophagy of cytosolic proteins by late endosomes. Dev. Cell 20, 131-139. doi: 10.1016/j.devcel.2010.12.003

Saman, S., Kim, W., Raya, M., Visnick, Y., Miro, S., Jackson, B., et al. (2011). Exosome-associated tau is secreted in tauopathy models and is selectively phosphorylated in cerebrospinal fluid in early alzheimer disease. J. Biol. Chem. 287, 3842-3849. doi: 10.1074/jbc.M111.277061

Sanders, D. W., Kaufman, S. K., DeVos, S. L., Sharma, A. M., Mirbaha, H., Li, A., et al. (2014). Distinct tau prion strains propagate in cells and mice and define different tauopathies. Neuron 82, 1271-1288. doi: 10.1016/j.neuron.2014. 04.047

Sardiello, M., Palmieri, M., Ronza, A., Di Medina, D. L., Valenza, M., Gennarino, V. A., et al. (2009). A gene network regulating lysosomal biogenesis and function. Science 325, 473-477. doi: 10.1126/science.1174447

Schaeffer, V., and Goedert, M. (2012). Stimulation of autophagy is neuroprotective in a mouse model of human tauopathy. Autophagy 8, 1686-1687. doi: 10.4161/ auto. 21488

Schelter, B. O., Shiells, H., Baddeley, T. C., Rubino, C. M., Ganesan, H., Hammel, J., et al. (2019). Concentration-dependent activity of hydromethylthionine on cognitive decline and brain atrophy in mild to moderate Alzheimer's disease. J. Alzheimers Dis. 72, 931-946. doi: 10.3233/JAD- 190772 
Schöll, M., Lockhart, S. N., Schonhaut, D. R., O’Neil, J. P., Janabi, M., Ossenkoppele, R., et al. (2016). PET imaging of tau deposition in the aging human brain. Neuron 89, 971-982. doi: 10.1016/j.neuron.2016.01.028

Settembre, C., Di Malta, C., Polito, V. A., Arencibia, M. G., Vetrini, F., Erdin, S., et al. (2011). TFEB links autophagy to lysosomal biogenesis. Science 332, 1429-1433. doi: 10.1126/science. 1204592

Shiells, H., Schelter, B. O., Bentham, P., Baddeley, T. C., Rubino, C. M., Ganesan, H., et al. (2020). Concentration-dependent activity of hydromethylthionine on clinical decline and brain atrophy in a randomized controlled trial in behavioral variant frontotemporal dementia. J. Alzheimers Dis. JAD 75, 501-519. doi: 10.3233/JAD- 191173

Shimada, K., Motoi, Y., Ishiguro, K., Kambe, T., Matsumoto, S. E., Itaya, M., et al. (2012). Long-term oral lithium treatment attenuates motor disturbance in tauopathy model mice: implications of autophagy promotion. Neurobiol. Dis. 46, 101-108. doi: 10.1016/j.nbd.2011.12.050

Skibinski, G., Parkinson, N. J., Brown, J. M., Chakrabarti, L., Lloyd, S. L., Hummerich, H., et al. (2005). Mutations in the endosomal ESCRTIII-complex subunit CHMP2B in frontotemporal dementia. Nat. Genet. 37, 806-808. doi: $10.1038 /$ ng1609

Song, J., Malampati, S., Zeng, Y., Durairajan, S. S. K., Yang, C., Tong, B. C., et al. (2020). A small molecule transcription factor EB activator ameliorates betaamyloid precursor protein and Tau pathology in Alzheimer's disease models. Aging Cell 19:e13069. doi: 10.1111/acel.13069

Tardivel, M., Bégard, S., Bousset, L., Dujardin, S., Coens, A., Melki, R., et al. (2016). Tunneling nanotube (TNT)-mediated neuron-to neuron transfer of pathological Tau protein assemblies. Acta Neuropathol. Commun. 4:117. doi: 10.1186/s40478-016-0386-4

Thorburn, J., Horita, H., Redzic, J., Hansen, K., Frankel, A. E., and Thorburn, A. (2009). Autophagy regulates selective HMGB1 release in tumor cells that are destined to die. Cell Death Diff. 16, 175-183. doi: 10.1038/cdd.2008.143

Trojanowski, J. Q., and Lee, V. M. Y. (2005). Pathological tau: a loss of normal function or a gain in toxicity? Nat. Neurosci. 8, 1136-1137. doi: 10.1038/ nn0905-1136

Uemura, N., Uemura, M. T., Luk, K. C., Lee, V. M. Y., and Trojanowski, J. Q. (2020). Cell-to-cell transmission of tau and $\alpha$-synuclein. Trends Mol. Med. 26, 936-952. doi: 10.1016/j.molmed.2020.03.012

Uytterhoeven, V., Deaulmerie, L., and Verstreken, P. (2018). Increased endosomal microautophagy reduces Tau driven synaptic dysfunction. Front. Neurosci. 12:102. doi: 10.3389/conf.fnins.2018.95.00102

Uytterhoeven, V., Lauwers, E., Maes, I., Miskiewicz, K., Melo, M. N., Swerts, J., et al. (2015). Hsc70-4 deforms membranes to promote synaptic protein turnover by endosomal microautophagy. Neuron 88, 735-748. doi: 10.1016/j.neuron.2015. 10.012

Van Acker, Z. P., Bretou, M., and Annaert, W. (2019). Endo-lysosomal dysregulations and late-onset Alzheimer's disease: impact of genetic risk factors. Mol. Neurodegen. 14:20. doi: 10.1186/s13024-019-0323-7

Vaz-Silva, J., Gomes, P., Jin, Q., Zhu, M., Zhuravleva, V., Quintremil, S., et al. (2018). Endolysosomal degradation of Tau and its role in glucocorticoid-driven hippocampal malfunction. EMBO J. 37:e99084. doi: 10.15252/embj.201899084

Wang, H., Wang, R., Carrera, I., Xu, S., and Lakshmana, M. K. (2016). TFEB overexpression in the P301S model of tauopathy mitigates increased PHF1 levels and lipofuscin puncta and rescues memory deficits. eNeuro 3, 9340-9351. doi: 10.1523/ENEURO.0042-16.2016
Wang, Y., Balaji, V., Kaniyappan, S., Krüger, L., Irsen, S., Tepper, K., et al. (2017). The release and trans-synaptic transmission of Tau via exosomes. Mol. Neurodegen. 12:5. doi: 10.1186/s13024-016-0143-y

Wang, Y., and Mandelkow, E. (2012). Degradation of tau protein by autophagy and proteasomal pathways. Biochem. Soc. Trans. 40, 644-652. doi: 10.1042/ BST20120071

Wang, Y., Martinez-Vicente, M., Krüger, U., Kaushik, S., Wong, E., Mandelkow, E.-M., et al. (2009). Tau fragmentation, aggregation and clearance: the dual role of lysosomal processing. Hum. Mol. Genet. 18, 4153-4170. doi: 10.1093/hmg/ ddp367

Wilcock, G. K., Gauthier, S., Frisoni, G. B., Jia, J., Hardlund, J. H., Moebius, H. J., et al. (2018). Potential of low dose leuco-methylthioninium Bis(Hydromethanesulphonate) (LMTM) monotherapy for treatment of mild Alzheimer's disease: cohort analysis as modified primary outcome in a phase III clinical trial. J. Alzheimers Dis. 61, 435-457. doi: 10.3233/JAD-170560

World Alzheimer Report (2019). World Alzheimer Report 2019: Attitudes to dementia; World Alzheimer Report 2019: Attitudes to Dementia. Available at: http:// www.daviddesigns.co.uk (accessed July 21, 2020).

Wu, J. W., Herman, M., Liu, L., Simoes, S., Acker, C. M., Figueroa, H., et al. (2013). Small misfolded tau species are internalized via bulk endocytosis and anterogradely and retrogradely transported in neurons. J. Biol. Chem. 288, 1856-1870. doi: 10.1074/jbc.M112.394528

Wu, J. W., Hussaini, S. A., Bastille, I. M., Rodriguez, G. A., Mrejeru, A., Rilett, K., et al. (2016). Neuronal activity enhances tau propagation and tau pathology in vivo. Nat. Neurosci. 19, 1085-1092. doi: 10.1038/nn.4328

Yamada, K., Holth, J. K., Liao, F., Stewart, F. R., Mahan, T. E., Jiang, H., et al. (2014). Neuronal activity regulates extracellular tau in vivo. J. Exp. Med. 211, 387-393. doi: $10.1084 /$ jem.20131685

Yang, X., and Tohda, C. (2018). Heat shock cognate 70 inhibitor, VER155008 , reduces memory deficits and axonal degeneration in a mouse model of Alzheimer's disease. Front. Pharmacol. 9:48. doi: 10.3389/fphar.2018. 00048

Yuan, A., Kumar, A., Peterhoff, C., Duff, K., and Nixon, R. A. (2008). Axonal transport rates in vivo are unaffected by tau deletion or overexpression in mice. J. Neurosci. 28, 1682-1687. doi: 10.1523/JNEUROSCI.5242-07.2008

Zhang, W., Falcon, B., Murzin, A. G., Fan, J., Crowther, R. A., Goedert, M., et al. (2019). Heparin-induced tau filaments are polymorphic and differ from those in alzheimer's and pick's diseases. eLife 8:e43584. doi: 10.7554/eLife.4 3584

Zhang, W., Tarutani, A., Newell, K. L., Murzin, A. G., Matsubara, T., Falcon, B., et al. (2020). Novel tau filament fold in corticobasal degeneration. Nature 580, 283-287. doi: 10.1038/s41586-020-2043-0

Conflict of Interest: The authors declare that the research was conducted in the absence of any commercial or financial relationships that could be construed as a potential conflict of interest.

Copyright (c) 2020 Jiang and Bhaskar. This is an open-access article distributed under the terms of the Creative Commons Attribution License (CC BY). The use, distribution or reproduction in other forums is permitted, provided the original author(s) and the copyright owner(s) are credited and that the original publication in this journal is cited, in accordance with accepted academic practice. No use, distribution or reproduction is permitted which does not comply with these terms. 